Cochrane Database of Systematic Reviews

\title{
Interventions for preventing distal intestinal obstruction syndrome (DIOS) in cystic fibrosis (Review)
}

Green J, Gilchrist FJ, Carroll W

Green J, Gilchrist FJ, Carroll W.

Interventions for preventing distal intestinal obstruction syndrome (DIOS) in cystic fibrosis.

Cochrane Database of Systematic Reviews 2018, Issue 6. Art. No.: CD012619.

DOI: 10.1002/14651858.CD012619.pub2.

www.cochranelibrary.com 
TABLE OF CONTENTS

HEADER 1

ABSTRACT

PLAIN LANGUAGE SUMMARY

SUMMARY OF FINDINGS

BACKGROUND

OBJECTIVES

METHODS

Figure 1.

RESULTS

Figure 2.

DISCUSSION

AUTHORS' CONCLUSIONS

ACKNOWLEDGEMENTS

REFERENCES

CHARACTERISTICS OF STUDIES

DATA AND ANALYSES

Analysis 1.1. Comparison 1 Cisapride versus placebo, Outcome 1 Total gastrointestinal symptoms.

Analysis 1.2. Comparison 1 Cisapride versus placebo, Outcome 2 Abdominal pain.

Analysis 1.3. Comparison 1 Cisapride versus placebo, Outcome 3 Abdominal distension. 
[Intervention Review]

\title{
Interventions for preventing distal intestinal obstruction syndrome (DIOS) in cystic fibrosis
}

\author{
Jessica Green ${ }^{1}$, Francis J Gilchrist ${ }^{1}$, Will Carroll²
}

1Academic Department of Child Health, Royal Stoke University Hospital, Stoke-on-Trent, UK. 2Department of Paediatric Respiratory

Medicine, University Hospitals of the North Midlands, Stoke-on-Trent, UK

Contact address: Will Carroll, Department of Paediatric Respiratory Medicine, University Hospitals of the North Midlands, Newcastle Road, Stoke-on-Trent, ST4 6QG, UK. will.carroll@nhs.net.

Editorial group: Cochrane Cystic Fibrosis and Genetic Disorders Group. Publication status and date: New, published in Issue 6, 2018.

Citation: Green J, Gilchrist FJ, Carroll W. Interventions for preventing distal intestinal obstruction syndrome (DIOS) in cystic fibrosis. Cochrane Database of Systematic Reviews 2018, Issue 6. Art. No.: CD012619. DOI: 10.1002/14651858.CD012619.pub2.

Copyright (c) 2018 The Cochrane Collaboration. Published by John Wiley \& Sons, Ltd.

\section{A B S T R A C T}

\section{Background}

Cystic fibrosis (CF) is the most common, life-limiting, genetically inherited disease. It affects multiple organs, particularly the respiratory system. However, gastrointestinal problems such as constipation and distal intestinal obstruction syndrome (DIOS) are also important and well-recognised complications in CF. They share similar symptoms e.g. bloating, abdominal pain, but are distinct conditions. Constipation occurs when there is gradual faecal impaction of the colon, but DIOS occurs when there is an accumulation of faeces and sticky mucus, forming a mass in the distal part of the small intestine. The mass may partially block the intestine (incomplete DIOS) or completely block the intestine (complete DIOS). Symptoms of DIOS can affect quality of life and other aspects of CF health, such as airway clearance, exercise, sleep and nutritional status. Treatment of constipation and prevention of complete bowel obstruction are required for gastrointestinal management in CF. However, many different strategies are used in clinical practice and there is a lack of consensus. The importance of this topic was highlighted in a recent research priority setting exercise by the James Lind Alliance.

\section{Objectives}

To evaluate the effectiveness and safety of laxative agents of differing types for preventing DIOS (complete and incomplete) in children and adults with CF.

\section{Search methods}

We searched the Cochrane Cystic Fibrosis and Genetic Disorders Group Trials Register comprising references identified from comprehensive electronic database searches and handsearches of relevant journals and abstract books of conference proceedings. Date of search: 22 May 2018.

We also searched online trial registries. Date of last search: 10 June 2018.

\section{Selection criteria}

Randomised and quasi-randomised controlled parallel trials comparing laxative therapy for preventing DIOS (including osmotic agents, stimulants, mucolytics and substances with more than one action) at any dose to placebo, no treatment or an alternative laxative therapy, in people of any age with pancreatic sufficient or insufficient CF and any stage of lung disease. Randomised cross-over trials were judged on an individual basis. 


\section{Data collection and analysis}

Two authors independently assessed trials for inclusion, extracted outcome data and performed a risk of bias assessment for the included data. We judged the quality of the evidence using GRADE criteria.

\section{Main results}

We included one cross-over trial (17 participants) with a duration of 12 months, in which participants were randomly allocated to either cisapride (a gastro-prokinetic agent) or placebo for six months each. The trial had an unclear risk of bias for most domains but had a high risk of reporting bias.

Radiograph scores revealed no difference in occurrence of DIOS between cisapride and placebo (narrative report, no data provided). There were no adverse effects. Symptom scores were the only secondary outcome within the review that were reported. Total gastrointestinal symptom scores favoured cisapride with a statistically significant mean difference (MD) of -7.60 (95\% confidence interval (Cl) -14.73 to -0.47). There was no significant difference at six months between cisapride and placebo for abdominal distension, $\mathrm{MD}-0.90$ (95\% $\mathrm{Cl}-2.39$ to 0.59) or abdominal pain, MD -0.4 (95\% Cl -2.05 to 1.25). The global symptom scores (whether individuals felt better or worse) were reported in the paper to favour cisapride and be statistically significant $(P<0.05)$.

We assessed the available data to be very low quality. There was a great deal of missing data from the included trial and the investigators failed to report numerical data for many outcomes. The overall risk of bias of the trial was unclear and it had a high risk for reporting bias. There was also indirectness; the trial drug (cisapride) has since been removed from the market in several countries due to adverse effects, thus it has no current applicability for preventing DIOS. The included trial also had very few participants, which downgraded the quality a further level for precision.

\section{Authors' conclusions}

There is an absence of evidence for interventions for the prevention of DIOS. As there was only one included trial, we could not perform a meta-analysis of the data. Furthermore, the included trial compared a prokinetic agent (cisapride) that is no longer licensed for use in a number of countries due to the risk of serious cardiac events, a finding that came to light after the trial was conducted. Therefore, the limited findings from the trial are not applicable in current clinical practice.

Overall, a great deal more research needs to be undertaken on gastrointestinal complications in CF, as this is a very poorly studied area compared to respiratory complications in CF.

\section{PLAIN LANGUAGE SUMMARY}

\section{Which interventions are effective and safe for preventing distal intestinal obstruction syndrome (DIOS) in cystic fibrosis?}

\section{Background}

Cystic fibrosis (CF) is an inherited, life-long condition that causes organ systems in the body to produce large amounts of thick and sticky mucus. The most commonly affected area is the lungs, in which thick mucus leads to recurrent chest infections and breathing difficulties. Another commonly affected area is the digestive system. Many people with CF suffer from bloating and abdominal pain which may be caused by constipation or distal intestinal obstruction syndrome (DIOS). In DIOS, overproduction of thick mucus combines with stool and sticks to the intestinal wall. This mass can partially block the intestine (incomplete DIOS) or completely block the intestine (complete DIOS). The latter causes severe pain, vomiting and is treated as a medical emergency. As part of effective care for people with CF, constipation should therefore be treated and complete bowel obstruction be prevented. It is also important to recognise that constipation and DIOS impact on other aspects of CF health. Bloating, abdominal pain and nausea may affect airway clearance, exercise and sleep. Nutritional status may also be affected due to decreased appetite and malabsorption. DIOS may affect the absorption of other medications taken by people with CF. Overall, DIOS can significantly impair quality of life. Different laxatives are currently used in clinics, but prescribing practices differ and there is no consensus on optimal treatment strategies. Hence, this review aimed to analyse the evidence for the preventing DIOS.

\section{Search date}

We last searched for evidence: 10 June 2018.

\section{Trial characteristics}

We included one trial in the review, which included 17 people aged between 13 to 35 years. These people were randomly put into groups to take either a placebo drug (with no active medication) or cisapride for six months each and then to cross over and take the alternative treatment for a further six months.

\section{Key results}

The trial used radiography to diagnose DIOS, but did not provide any data and only stated that there was no difference between cisapride and placebo. The trial also stated that there were no adverse effects from the cisapride. The trial assessed participant-reported total and 
individual gastrointestinal symptom scores. People in the cisapride group reported an improvement in total gastrointestinal symptom scores compared to those in the placebo group. However, there were no differences reported between groups for the individual symptom scores of abdominal pain and abdominal distension (swelling). Participants also reported global symptom scores, which showed that most people felt better taking cisapride compared to placebo.

\section{Quality of the evidence}

The overall quality of the evidence was very low. With one trial in this review, we could not combine data from different trials. The trial did not provide enough information about the methods used for allocating participants or about missing data and did not fully report certain results. The small number of participants also lowered the precision of the results. Since this trial was conducted, cisapride has been removed from the market in a number of countries due to rare but serious heart complications, therefore it has no applicability to current clinical practice. 


\section{SUMMARY OF FINDINGS}

Summary of findings for the main comparison. Cisapride compared to placebo for preventing distal intestinal obstruction syndrome (DIOS) in cystic fibrosis

\section{Cisapride compared to placebo for preventing distal intestinal obstruction syndrome (DIOS) in cystic fibrosis}

Patient or population: preventing DIOS in cystic fibrosis

Setting: tertiary Centre

Intervention: cisapride

Comparison: placebo

\begin{tabular}{|c|c|c|c|c|c|c|}
\hline \multirow[t]{2}{*}{ Outcomes } & \multicolumn{2}{|c|}{ Anticipated absolute effects ${ }^{\star}(95 \% \mathrm{Cl})$} & \multirow{2}{*}{$\begin{array}{l}\text { Relative effect } \\
(95 \% \mathrm{Cl})\end{array}$} & \multirow{2}{*}{$\begin{array}{l}\text { № of partici- } \\
\text { pants } \\
\text { (studies) }\end{array}$} & \multirow{2}{*}{$\begin{array}{l}\text { Certainty of } \\
\text { the evidence } \\
\text { (GRADE) }\end{array}$} & \multirow[t]{2}{*}{ Comments } \\
\hline & Risk with placebo & Risk with cisapride & & & & \\
\hline \multicolumn{7}{|l|}{ Follow-up: baseline to 6 months } \\
\hline $\begin{array}{l}\text { Adverse effects (participant inter- } \\
\text { views) }\end{array}$ & $\begin{array}{l}\text { No adverse effects } \\
\text { group. }\end{array}$ & e noted in either & NA & $\begin{array}{l}17 \\
(1 \mathrm{RCT})\end{array}$ & $\begin{array}{l}\oplus \ominus \ominus \ominus \\
\text { VERY LOW } 234\end{array}$ & $\begin{array}{l}\text { No numerical data avail- } \\
\text { able. }\end{array}$ \\
\hline \multicolumn{7}{|l|}{ Follow-up: 3 to 12 months } \\
\hline $\begin{array}{l}\text { Total gastrointestinal symptom } \\
\text { scores (participant-reported } \\
\text { symptom scores from } 20 \text { to } 100 \text { ) } \\
\text { Follow-up: } 3 \text { to } 12 \text { months }\end{array}$ & \multicolumn{2}{|c|}{$\begin{array}{l}\text { The mean difference was } 7.6 \text { lower in the cis- } \\
\text { apride arm } \\
\text { ( } 14.73 \text { lower to } 0.47 \text { lower) than when the } \\
\text { same participants took a placebo. }\end{array}$} & NA & $\begin{array}{l}17 \\
(1 \mathrm{RCT})\end{array}$ & $\begin{array}{l}\oplus \ominus \ominus \ominus \\
\text { VERY LOW } 234\end{array}$ & $\begin{array}{l}\text { Score made up of } 10 \text { differ- } \\
\text { ent gastrointestinal symp- } \\
\text { toms: heartburn, flatulence, } \\
\text { regurgitation, fullness, ab- } \\
\text { dominal distension, abdom- } \\
\text { inal pain, diarrhoea, nausea, } \\
\text { vomiting, anorexia. }\end{array}$ \\
\hline Hospitalisation for any cause & \multicolumn{3}{|c|}{ Outcome not reported. } & NA & NA & \\
\hline Hospitalisation for DIOS & \multicolumn{3}{|c|}{ Outcome not reported. } & NA & NA & \\
\hline Quality of life & \multicolumn{3}{|c|}{ Outcome not reported. } & NA & NA & \\
\hline Tolerability & \multicolumn{3}{|c|}{ Outcome not reported. } & NA & NA & \\
\hline
\end{tabular}

${ }^{*}$ The risk in the intervention group (and its $95 \% \mathrm{Cl}$ ) is based on the assumed risk in the comparison group and the relative effect of the intervention (and its $95 \% \mathrm{Cl}$ ). 
GRADE Working Group grades of evidence

High certainty: we are very confident that the true effect lies close to that of the estimate of the effect.

Moderate certainty: we are moderately confident in the effect estimate: The true effect is likely to be close to the estimate of the effect, but there is a possibility that it is substantially different.

Low certainty: our confidence in the effect estimate is limited: The true effect may be substantially different from the estimate of the effect.

Very low certainty: we have very little confidence in the effect estimate: The true effect is likely to be substantially different from the estimate of effect.

1. Selective reporting may have occurred with this outcome; allocation concealment and sequence generation was unclear.

2. Cisapride is a prokinetic, not a typical laxative agent (different to protocol). The study was conducted in 1990 when cisapride was still prescribed. It has now been taken off the

UK market and other international markets due to its rare but serious cardiac effects.

3. Very small number of participants in the trial does not give sufficient information to give a precise effect estimate.

4. Allocation concealment and sequence generation ranked as unclear risk of bias. 


\section{B A C K G R O U N D}

Cystic fibrosis (CF) is an important genetic disorder. It is life-limiting and affected individuals have dysfunction of several organ systems which results in morbidity and reduced quality of life (QoL). To be affected a person must possess two faulty copies of the gene that encodes a protein called the cystic fibrosis transmembrane conductance regulator (CFTR). About one in 25 of the UK white population carry a single faulty copy of this gene and one in 2500 newborns in the UK are born with CF (Tobias 2011). Worldwide the condition affects approximately 70,000 children and adults (CF Foundation 2016).

Although respiratory symptoms are prominent, and often the focus of clinical care, CF is a multifaceted disease which also has important effects on the gastrointestinal and endocrine systems. Effective management of $\mathrm{CF}$ is made more challenging by the fact that problems in any of these systems can interact in an adverse way. The CFTR is expressed in many cell types throughout the body; it regulates chloride transport and thus indirectly influences water transport across the cell membranes. Absent or dysfunctional CFTR leads to thickened, dehydrated mucus.

\section{Description of the condition}

Distal intestinal obstruction syndrome (DIOS) is a well-recognised morbidity in CF. It is the result of the accumulation of thick and sticky material within the bowel (both mucus and faeces) particularly in the final part of the small intestine (the terminal ileum and caecum). This mass becomes connected to the bowel wall itself and the finger-like projections of the small bowel (intestinal villi) making it fixed in position and difficult to remove (Colombo 2011). The bowel may be completely blocked (complete DIOS) or only partially blocked (incomplete DIOS), e.g. when a persistent mass is found low down on the right-hand side (right iliac fossa). Previously DIOS was known as meconium ileus equivalent (MIE), and affects between $10 \%$ to $22 \%$ of individuals with CF (Davidson 1987; Dray 2004; Penketh 1987; Rubinstein 1986). It affects adults more than children; the estimated prevalence of DIOS is between 5 and 12 episodes per 1000 patient years for children, but 35.5 per 1000 patient years for adults (Anderson 1990; Colombo 2011; Houwen 2010). Once an individual has had DIOS the recurrence risk is approximately 50\% (Dray 2004). A number of factors contribute to the occurrence of DIOS. It occurs more commonly in individuals who have pancreatic enzyme deficiency (Munck 2016); less than 10\% of individuals with DIOS are pancreatic sufficient (Houwen 2010). Anecdotally, it is also more common in those who do not adhere to pancreatic enzyme replacement therapy. Meconium ileus (a type of bowel obstruction occurring shortly after birth) is another recognised risk factor, with up to $50 \%$ of those with DIOS having been affected by this (Houwen 2010). The incidence of DIOS may also be increased after transplantation surgery and can be as high as 20\% (Gilljam 2003; Millar-Jones 1995). In part, it occurs due to the loss of CFTR function in the intestine, where CFTR regulates chloride, bicarbonate and sodium transport.

\section{Distinguishing DIOS from other causes of bowel obstruction in CF}

The CF gut is prone to obstruction from other causes due to its altered pathophysiology (Van der Doef 2011). A small but significant proportion of newborns with CF present either at birth or shortly afterwards with bowel obstruction - meconium ileus.
Meconium ileus occurs in $13 \%$ to $17 \%$ of the CF population (Van der Doef 2011). Throughout life, children and adults with CF are prone to constipation, with almost half of all children studied (47\%) having evidence of constipation (Van der Doef 2010). However, it is possible to distinguish between constipation and DIOS both clinically and radiologically. One widely-used definition of DIOS is an acute complete or incomplete faecal obstruction in the ileocecum; whereas constipation is defined as gradual faecal impaction of the total colon (Houwen 2010). Individuals are more likely to describe an exact time when their symptoms started in the acute picture of DIOS, but will describe a more chronic build up of symptoms in constipation. It is therefore important to rule out other surgical diagnoses when considering DIOS, such as intussusception, volvulus and strangulated hernia. Using this definition of DIOS in individuals under 18 years of age, 51 episodes in 39 individuals were recorded, giving an overall incidence of 6.2 (95\% confidence interval $(\mathrm{Cl}) 4.4$ to 7.9 ) episodes per 1000 patientyears. Although there is undoubtedly overlap between constipation and incomplete DIOS, the clinical definition proposed by Houwen permits the effectiveness of treatments to be monitored clinically (Houwen 2010). It is worth noting that prior to this definition of DIOS (incomplete versus complete) in 2010 , studies may not have accurately reported the incidence of DIOS due to to some episodes of constipation being considered as incomplete DIOS. In this light, one should consider that although previous studies have estimated different incidences for DIOS between children and adults, Munck found similar incidences between these two groups in a more recent study (Munck 2016).

\section{Description of the intervention}

Treatment of constipation and the prevention of complete bowel obstruction is required as part of optimal care for individuals with CF. DIOS is predominantly an ileocaecal pathology (Houwen 2010). Many strategies are currently used in clinical practice and there is a lack of consensus about what the best preventative measures are likely to be. In addition to ensuring adequate hydration and adherence with pancreatic enzyme supplementation, different centres use different combinations of laxatives to prevent DIOS including lactulose, senna, polyethylene glycol (e.g. Movicol ${ }^{\circledR}$ ), sodium docusate, sodium picosulphate and fibre.

Although most children and adults with $\mathrm{CF}$ are prescribed interventions to prevent DIOS at some stage, there is significant heterogeneity observed between clinicians in their choices of agent. With the advent of newer laxative agents, e.g. Movicol ${ }^{\circledR}$, some centres have changed their approach.

This review focuses upon the use of laxative agents (aperients) for preventing DIOS. There are three main groups of laxatives based upon their primary mechanism of action (although there is overlap between the mechanism of action for some agents). Aside from the main groups of laxative, prokinetic agents may also prevent DIOS, although these are less commonly used in clinical practice (post hoc change).

\section{Osmotic laxatives}

Osmotic laxatives are faecal softeners which work by increasing water in the large bowel, either by drawing fluid from the body into the bowel or by retaining the fluid they were administered with. 


\section{Lactulose}

Lactulose is given orally; it is widely used, but may cause flatulence or abdominal pain in high doses (Colombo 2011).

\section{Macrogol 3350}

Macrogol 3350 is also known as polyethylene glycol, or under the brand names Movicol $^{\circledast}$, Laxido ${ }^{\circledR}$ or Klean-Prep ${ }^{\circledR}$. Movicol ${ }^{\circledR}$ is recommended as first-line treatment for constipation (NICE 2015). It is commonly given to children for chronic constipation or at a higher dose in faecal impaction. It can be given as an oral solution or powder (BNFc 2016). Laxido ${ }^{\circledR}$ is a very similar product which is also recommended for treatment of chronic constipation or impaction. Klean-Prep ${ }^{\circledR}$ can also be used, with the aim to cleanse the bowel. The solution is given until clear fluid is passed per rectum. As larger volumes are required, it is often necessary to administer via gastrostomy or nasogastric tube (Colombo 2011; NICE 2015).

\section{Diatrizoate}

Oral diatrizoate (also known as Gastrografin ${ }^{\circledR}$ ) is used by many centres to treat DIOS. It is given as a single dose, which can be repeated after 24 hours. Rectal diatrizoate can also be used in more severe cases (Colombo 2011). As diatrizoate is highly osmotic, the individual must be adequately hydrated prior to administration in order to avoid complications such as shock and perforation of the bowel (Tuldahar 1999).

\section{Stimulant laxatives}

\section{Senna}

Senna acts by stimulating peristalsis and increases the emptying of the bowel. Senna is therefore useful when the individual has soft stools, but finds it difficult to pass them (NICE 2015).

\section{Sodium docusate}

Sodium docusate acts both as a stimulant and also as a stool softener. It can be administered orally, but if this does not relieve faecal impaction, the drug can also be given as an enema (NICE 2015).

\section{Sodium picosulphate}

Sodium picosulphate acts by stimulating the mucosa of the large bowel, increasing its motility; it is given as an oral solution (BNFC 2016).

\section{Mucolytics}

\section{Oral $\mathrm{N}$-acetylcysteine}

$\mathrm{N}$-acetylcysteine (also known as Parvolex ${ }^{\circledR}$ ) is indicated for abnormal or impaired mucus production. It can be given as a single oral dose for treatment of meconium ileus or DIOS. It is typically diluted in a sweet drink, such as orange juice or cola, to mask the strong and bitter taste (BNFc 2016).

\section{Prokinetics}

Aside from the main groups of laxatives already mentioned, we assessed the evidence for prokinetic agents in preventing DIOS (post hoc change). These agents work by increasing the co-ordination of gut wall contractions and motility, enhancing the displacement of bowel contents (Tonni 1996). Examples of prokinetic agents include erythromycin, metoclopramide, cisapride, renzapride, domperidone and octreotide. Three prokinetic agents (prucalopride, linaclotide and lubiprostone) are specifically recommended in the UK by NICE guidelines for refractory constipation syndromes (NICE 2017).

\section{How the intervention might work}

Different aperients have different mechanisms of action. Historically these have been divided into the broad categories described above. In clinical practice it has been helpful to titrate the doses of these to achieve a reduction in abdominal pains and a normal physical examination, e.g. resolution of right iliac fossa mass. Some newer agents (e.g. Movicol $^{\circledR}$ ) combine these effects providing both softening and stimulation.

For preventing DIOS, laxatives are likely to work by increasing stool volume and reducing gut transit time or by softening mucofaeculant material that has built up in the gut. The passage of larger volumes of more liquid stool may have a mechanical effect on any adherent mucofaeces. However, the use of high doses of laxatives are likely to lead to other undesirable consequences including the unacceptable frequency of stooling, soiling, abdominal distension, flatulence and abdominal pain.

\section{Why it is important to do this review}

Intestinal obstruction is an important and common problem in CF as highlighted by the recent research priority setting partnership with the James Lind Alliance (Rowbotham 2017). Incomplete DIOS is relatively common and there is considerable variation in practice. In our clinical experience, prophylaxis for DIOS is given to individuals who have had an episode of complete DIOS, those who have clinical signs consistent with incomplete DIOS or those with pancreatic insufficiency and clinical or radiological manifestations of constipation (e.g. faecal masses palpable on clinical examination or reported abdominal pain). The evidence base for this practice is unclear and there is no clear evidence base for any preventative therapies for DIOS (Colombo 2011).

Individuals with CF undergo a very large treatment burden. In discussing the risks and benefits of preventative treatment for DIOS it is important that we give clear information about the likely side effects and tolerability of any proposed therapy.

\section{O B JECT IVES}

To evaluate the effectiveness and safety of laxative agents of differing types for preventing DIOS (complete and incomplete) in children and adults with $\mathrm{CF}$.

\section{METHODS}

\section{Criteria for considering studies for this review}

\section{Types of studies}

We have included randomised controlled trials (RCTs) and quasiRCTs of parallel design. We assessed quasi-RCTs on their merit using the Cochrane risk of bias tool and if review authors were satisfied that the groups were similar at baseline, we included them.

We also assessed cross-over trials for possible inclusion on an individual basis. If we deemed the treatment to alter the condition to the extent that, on entry to subsequent phases, the participants 
differ from their initial state, we excluded the trial unless we could use data from the first phase only (see Unit of analysis issues).

\section{Types of participants}

Children and adults with CF diagnosed by sweat test or genetic testing, with all stages and severity of lung disease and with or without pancreatic sufficiency.

\section{Types of interventions}

We compared the different treatment groups of enteral laxative therapy for preventing DIOS (including osmotic agents, stimulants, mucolytics, substances which have more than one action and prokinetic agents (post hoc change)) at any dose to placebo, no treatment or an alternative oral laxative therapy.

As some treatments have significant overlap in their mechanisms of action (e.g. Movicol ${ }^{\circledR}$ is a osmotic agent which also has a stimulant effect), we planned to initially examine whether any preventative treatment is effective and then to examine the relative effectiveness of different classes of agents as a subgroup analysis.

\section{Types of outcome measures}

\section{Primary outcomes}

1. Complete or incomplete DIOS diagnosed with a clinical (e.g. right iliac fossa or distension or pain) and radiological (e.g. small intestinal dilated bowel or faecal mass) combination

2. Adverse effects from treatment

a. serious adverse effects of treatment regimens (including, but not limited to, rectal bleeding, intestinal perforation, mucosal erosions, anaphylactic reaction, vomiting with electrolyte disturbance)

b. other adverse effects of treatment (e.g. diarrhoea or soiling, abdominal distension, loss of continence or pain)

\section{Secondary outcomes}

1. Time to hospital admission
a. all causes

b. due to DIOS

2. Participant-reported QoL scores

3. Participant-reported symptom scores

4. Tolerability (participant- or investigator-reported rates of concordance)

\section{Search methods for identification of studies}

We searched for all relevant published and unpublished trials without restrictions on language, year or publication status.

\section{Electronic searches}

We identified relevant studies from the Group's Cystic Fibrosis Trials Register using the terms: distal intestinal obstruction syndrome [DIOS].

The Cystic Fibrosis Trials Register is compiled from electronic searches of the Cochrane Central Register of Controlled Trials (CENTRAL) (updated each new issue of the Cochrane Library), weekly searches of MEDLINE, a search of Embase to 1995 and the prospective handsearching of two journals - Pediatric Pulmonology and the Journal of Cystic Fibrosis. Unpublished work is identified by searching the abstract books of three major cystic fibrosis conferences: the International Cystic Fibrosis Conference; the European Cystic Fibrosis Conference and the North American Cystic Fibrosis Conference. For full details of all searching activities for the register, please see the relevant sections of the Cochrane Cystic Fibrosis and Genetic Disorders Group website.

Date of last search: 22 May 2018.

We searched the following databases between 09 May 2018 and 10 June 2018:

- Cochrane Central Register of Controlled Trials (CENTRAL; 2017, Issue 12) in the Cochrane Library (www.thecochranelibrary.com) (searched 09 May 2018);

- MEDLINE Ovid (1946 to 09 May 2018);

- Embase Ovid (1974 to 10 June 2018).

We also searched the following trials registries and other resources between 09 May 2018 and 16 May 2018:

- US National Institutes of Health Ongoing Trials Register Clinicaltrials.gov (www.clinicaltrials.gov; searched 16 May 2018);

- International Standard Randomised Controlled Trial Number (ISRCTN) Registry (www.isrctn.com; searched 09 May 2018);

- World Health Organization International Clinical Trials Registry Platform (WHO ICTRP) (apps.who.int/trialsearch; searched 16 May 2018);

- Open Grey (www.opengrey.eu/; searched 09 May 2018).

For details of our search strategies, please see the appendices (Appendix 1).

\section{Searching other resources}

We checked the bibliographies of included trials and any relevant systematic reviews identified for further references to relevant trials.

\section{Data collection and analysis}

\section{Selection of studies}

Once we had the complete list of identified references, one author (JG) checked for and removed any duplicates. Two authors (WC and JG) then reviewed all titles and abstracts and discarded references which clearly did not meet the inclusion criteria. We attempted to resolve any disagreements by discussion, but if we could not reach a decision, we asked the third author (FG) of the review to mediate until we reached a final decision. Once we discarded trials on the basis of title and abstract, we obtained full copies of the remaining references and screened these using a standardised screening form customised for this review.

We considered trials in any language and translated them as necessary. We included trials published as full texts, but if there was only an abstract available, we included it if it presented results. If there were no results available within the abstract or on any trials registry sites, then we classified the trial as 'Studies awaiting assessment' until more information is available. Similarly with unpublished trials, if a trial met our inclusion criteria and quality assessment then we included it. 
We presented the results of the search using a standardised flow chart (Figure 1). 
Figure 1. Study flow diagram (Moher 2009)

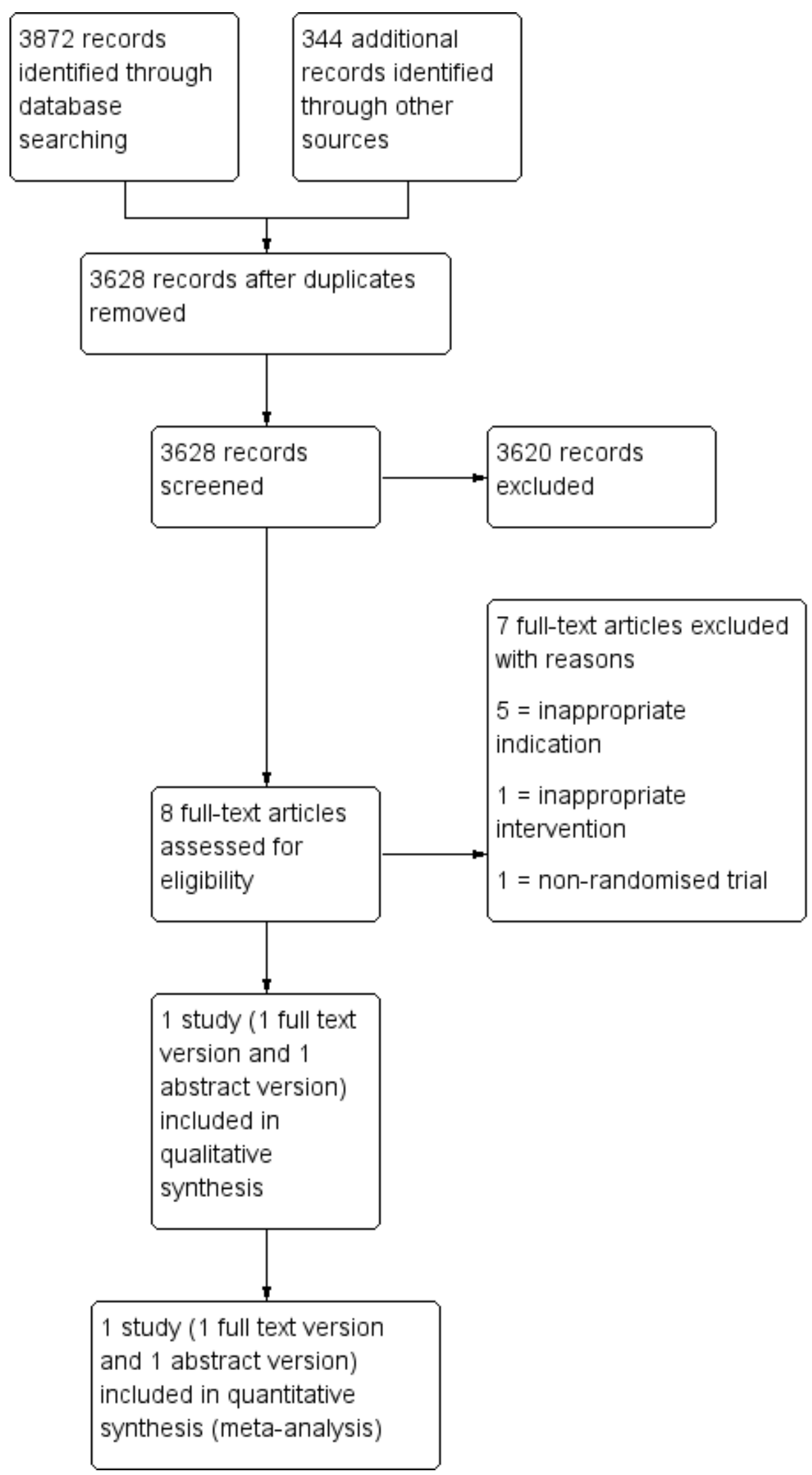




\section{Data extraction and management}

Two authors (WC and JG) independently extracted data using a specially designed data extraction form developed by the Cochrane Cystic Fibrosis and Genetic Disorders Review Group and adapted to this review. We collected data on:

\section{- participant characteristics;}

- trial characteristics and trial design;

- intervention and comparator;

- outcome data - we will report data for each outcome separately.

One author (WC) checked the independent data extraction forms for discrepancies and if there were any which we could not resolve by discussion, a third author (FG) arbitrated.

We entered the extracted data into the Review Manager software for analysis (RevMan 2014). We planned to initially carry out a comparison of any laxative agent versus placebo or usual treatment and then, if possible, undertake a subgroup analysis by type of laxative (see Subgroup analysis and investigation of heterogeneity), but we did not do this as we only included one trial in the review.

\section{Assessment of risk of bias in included studies}

We used the risk of bias tool as described in the Cochrane Handbook for Systematic Reviews of Interventions to assess the risk of bias across six domains (sequence generation, allocation concealment, blinding, incomplete outcome data, selective reporting and other potential sources of bias) (Higgins 2011).

We planned that if the trial described the methods of randomisation and allocation, including the concealment of the allocation sequence from the researchers, and we deemed these to be adequate, then we would rank the trial as having a low risk of bias for this domain. Where these were inadequate, we would rank the trial as being at a high risk and where it was unclear from the description given, then we would rank it as having an unclear risk of bias.

Similarly for blinding, we looked at the method used and who was blinded to determine the risk of bias.

We planned to extract information on missing data and how the investigators recorded participant withdrawals and loss to followup. We also planned to look at whether missing data were equally distributed between the intervention and control groups. If all review authors agreed that missing data had been accounted for adequately, then we would judge the trial to be at a low risk of bias. We would record the trial as having a high risk of bias if investigators did not adequately report the missing data and would record it as having an unclear risk of bias if we were unable to see how the missing data had been reported. Two authors assessed the included trial to determine whether the investigators used an intention-to-treat (ITT) analysis and again, once we reached an agreement, we determined the risk of bias as being high, low or unclear.

We planned that if the trial investigators reported all outcomes in the paper, we would record a low risk of bias from selective reporting. If the paper stated that investigators measured outcomes, but they did not report the results of these, we would rank the paper as being at high risk. If it was unclear to us whether the trial reported all outcomes measured, then we would state this and rank it as unclear for this domain. We planned to search for trial protocols to be able to assess outcome reporting. If we could not locate the protocol, we planned to assess outcome reporting based on a comparison between the methods section of the full published paper and the results section. As we could not locate the protocol of the included trial, this is how we assessed potential reporting bias.

The review authors looked for any other potential sources of bias in the included trial and recorded what they found. According to these findings, if neither author had found any other source of bias, then we would rank the trial as having a low risk for this domain and high risk if the opposite was true.

We presented the results of the risk of bias assessment both individually and in a summary table.

\section{Measures of treatment effect}

For dichotomous data (complete DIOS, incomplete DIOS, pooled adverse effects, failure to tolerate treatment and adherence), we planned to calculate a pooled estimate of the treatment effects for each outcome across trials using the risk ratio (RR) and 95\% confidence intervals (Cls) where appropriate. For individual adverse events, e.g. reported soiling, we planned to report $99 \% \mathrm{Cls}$.

For continuous data (participant-reported QoL, symptom scores) we planned to record the mean change and standard deviation (SD) from baseline for each group. We intended to calculate a pooled estimate of treatment effect using the mean difference (MD) and $95 \% \mathrm{Cls}$. Where trials used different units of measurement or measurement scales for reporting the same outcome (which is likely to be true for QoL and symptom scores) we planned to use the standardised mean difference (SMD) to report the results. Where trials only reported a pre-intervention mean (SD) and postintervention mean (SD) then we planned to calculate the mean change but not the SD of the change. We would report these results narratively. For the included trial we used the generic inverse variance method to generate a MD and corresponding $95 \% \mathrm{Cls}$ due to limitations of the available data.

For time-to-event data (e.g. time to hospitalisation) we intended to express the intervention effect as a hazard ratio (HR) with $95 \% \mathrm{Cls}$ using the generic inverse variance method.

Where end-points are semantically different but report similar outcomes then we planned to group outcomes. Thus, synonymous terms are considered jointly. We considered:

- abdominal distension (reported) to be synonymous with bloating, swelling or gaseous distension;

- pain to be synonymous with discomfort or ache;

- vomiting to be synonymous with emesis;

- constipation to be synonymous with straining or dyschezia.

\section{Unit of analysis issues}

We assessed the included trial, which is of cross-over design, to establish how much data we could include in the analysis. We planned that where the authors had taken account of the crossover design in the analysis, any carry-over effect and within-person differences, we would be able to include the trial. Where the data had not been analysed appropriately, we may have been able to include data from the first phase of the cross-over trial as if it were 
a parallel design; although the advantage of the cross-over design (using participants as their own controls) would be lost (Elbourne 2002). We were able to include both phases of the trial, as the authors accounted for the carry-over treatment effect and analysed the data whilst taking into account the cross-over design.

If we had found multi-arm trials which fall into more than one comparison, and where the two active treatment arms are different types of laxative regimen, e.g. Movicol ${ }^{\circledR}$ versus lactulose and senna versus placebo, we planned to analyse each treatment arm separately against placebo and where appropriate include results in a meta-analysis. If the two active treatment arms had been of the same type of laxative (e.g. softening agents), but employed a different laxative or dose, we would have combined them against the placebo arm to look at the effect of the type of laxative rather than an individual drug.

If there had been heterogeneity between trials looking at different types of laxative regimen, we planned to carry out a subgroup analysis to look at the effect of individual drugs (Subgroup analysis and investigation of heterogeneity).

\section{Dealing with missing data}

We attempted to request additional data from the trial author(s) if there were insufficient data in the published paper or uncertainty about data we were able to extract from the included trial. We planned to undertake an ITT analysis wherever possible throughout the review.

We also planned to assess the extent to which trial authors have employed an ITT analysis and we planned to report the numbers of participants who dropped out of each arm of the trial, where possible.

Where data were incomplete but partially available we planned to use the last available measurement to determine effectiveness.

\section{Assessment of heterogeneity}

If we had included trials reporting the same outcomes which we were able to include in a meta-analysis, we planned to assess the level of heterogeneity using the $\mathrm{I}^{2}$ statistic. We intended to look at the overlap of the Cls on the forest plots to gauge the significance of the $I^{2}$ value.

We planned to base our definitions of different levels of heterogeneity on those described in the Cochrane Handbook for Systematic Reviews of Interventions:

- low (might not be important) - $0 \%$ to $40 \%$;

- moderate - $30 \%$ to $60 \%$;

- substantial - $50 \%$ to $90 \%$; and

- considerable $-75 \%$ to $100 \%$.

The Cochrane Handbook for Systematic Reviews of Interventions states that this is a rough guide because the importance of inconsistency depends on several factors (Deeks 2011).

\section{Assessment of reporting biases}

Where we had been able to include at least 10 trials, we planned to generate a funnel plot to attempt to identify any publication bias in the included trials (Sterne 2011). We also planned to identify any selective reporting in the included publications, by comparing the trial protocols with the final papers and by careful examination of the trial publications and consideration of reporting of both positive and negative effects of the intervention. Where trial protocols were not available, we planned to compare the outcomes reported in the results section against the methods section of the paper. We planned to extract information on the sponsors, sources of funding and competing interests of the authors to determine the role of external bias being introduced. To minimise publication bias, we planned to search trial registries and contact pharmaceutical companies for unpublished data. For the included trial in the review, we did not have access to the protocol, so compared the methods section with the results section. We were also able to gather limited information sources of support for the trial, but did not on sources of funding or competing interests. We searched trial registries for unpublished data on the included trial, but could not find additional data.

\section{Data synthesis}

We were not able to combine trials in a meta-analysis, but if we are able to do so in future updates of this review we will use the data from the selected trials to generate forest plots using the Review Manager software (RevMan 2014). We will carry out an initial combined analysis of all types of laxative agent) followed by separate meta-analyses for different groups of laxative agents (e.g. osmotic laxatives, stimulants and those with a combined mechanism of action) and mucolytics. We will examine the level of heterogeneity to determine which type of analysis model to use. If there is low heterogeneity (less than $40 \%$ ) then we will use a fixedeffect model and if the $\mathrm{I}^{2}$ statistic is greater than $40 \%$ then we will use a random-effects model to summarise the data.

\section{Subgroup analysis and investigation of heterogeneity}

In future, if there is greater than $40 \%$ heterogeneity among the included trials, we will undertake subgroup analyses to look at the following:

- children (18 years and under) versus adults;

- type of laxative (osmotic agent (e.g. lactulose) versus stimulant laxative regimes (e.g. senna) versus mucolytic (e.g. Nacetylcysteine));

- single regimens versus combined regimens (e.g. lactulose and senna);

- effectiveness of regimen in preventing complete versus incomplete DIOS* (Houwen 2010).

*The following definitions of complete and incomplete DIOS are taken from (Houwen 2010).

1. Complete intestinal obstruction as evidenced by vomiting of bilious material and/or fluid levels in small intestine on an abdominal radiography.

2. Faecal mass in ileo-caecum.

3. Abdominal pain or distension (or both).

Complete DIOS is defined as when all three of the above criteria are present, whereas incomplete or impending DIOS is defined as only the second and third criteria being present.

\section{Sensitivity analysis}

If we are able to perform a meta-analysis in future updates, we will carry out sensitivity analyses to look at the effect of the risk of bias 
findings. We will look at the effect of adding in and taking out trials where there is high risk of bias. We will also attempt to examine the effect of cross-over trials on the results by carrying out a sensitivity analysis to include and exclude them.

\section{Summary of findings table}

We reported summary of findings information for the comparison of cisapride versus placebo. In future updates we will present a separate table for each treatment comparison, i.e. laxative agents versus control, placebo or alternate regimens. In the summary of findings table we assess our chosen outcomes: prevention of complete or incomplete DIOS, adverse events, hospitalisation for any cause, hospitalisation for DIOS, QoL, symptom score, and tolerability.

For each outcome we planned to report the illustrative risk with and without the intervention, magnitude of effect (RR or MD), numbers of trials and participants addressing each outcome and a grade of the overall quality of the body of evidence using the Grading of Recommendations Assessment, Development and Evaluation (GRADE) with comments (Schunemann 2006). We report the available data and where no data for individual outcomes were available, we state this in the table.

\section{RES U L T S}

\section{Description of studies}

\section{Results of the search}

Of the 2631 papers (after 588 duplicates were removed) identified by initial searches, we found eight potentially eligible trials for inclusion. Of these, one trial was included and seven were excluded. The PRISMA flow diagram illustrates this process (Figure 1).

\section{Included studies}

The included trial $(n=17)$ was available as an abstract and full text (Koletzko 1990). See the tables for more information (Characteristics of included studies).

\section{Trial design}

The trial was a randomised, double-blind, placebo-controlled cross-over trial based at a single centre in Toronto, Canada (Koletzko 1990).

The duration of the trial was 12 months. The participants were randomised into two groups to take either the placebo or active drug (cisapride) and then swapped to the other treatment group after six months. The trial accounted for any potential carryover treatment effect of the active drug by analysing the data in two ways. Measurements were recorded twice for each sixmonth period, once every three months. Firstly, investigators took an average of the two measurements, they then discarded the measurements from the first three months, to account for any cumulative effect of the drug.

\section{Participant characteristics}

In terms of the inclusion criteria, the trial required participants to have a diagnosis of CF and to have had one or more episodes of DIOS in the preceding 12 months. The trial excluded pregnant participants, those with current gastrointestinal obstruction and with serious cardiovascular, neurological, renal or hepatic disease. Participants with other causes of abdominal pain e.g. peptic ulcer disease and inflammatory bowel disease were also excluded, as were those who regularly used metoclopramide, domperidone or an anticholinergic drug.

There were 17 participants randomised, 12 males and five females. The recruiting age was not specified, but the mean (range) age in years of participants was 21.0 (12.9 to 34.9$)$ years. The baseline characteristics for participants were stated; there were no significant differences in the clinical characteristics between them at the start of each treatment period.

\section{Intervention}

Participants weighing between $40 \mathrm{~kg}$ and $50 \mathrm{~kg}$ received $7.5 \mathrm{mg}$ of either placebo or cisapride three times per day and those weighing over $50 \mathrm{~kg}$ received $10 \mathrm{mg}$ of either placebo or cisapride three times per day.

\section{Outcomes}

The radiological diagnosis of DIOS was measured in the trial using supine abdominal radiographs. Participants were interviewed for adverse effects and also reported any gastrointestinal and global symptoms, with the use of scoring systems. Other outcomes included the number of participants requiring therapy for DIOS and stool weight.

Outcomes reported in the trial but not presented in this review included anthropometric measurements (e.g. mid-arm circumference, skin fold thickness), frequency of pulmonary infections, pulmonary function ( $\left.\% \mathrm{FEV}_{1}\right)$, nutritional and calorie intake, routine laboratory tests (e.g. urinalysis, complete blood count) and number of hospital admissions.

\section{Excluded studies}

We excluded seven trials, please see the tables for more information (Characteristics of excluded studies).

Five trials were excluded because the active drug was not used to prevent DIOS and therefore reported irrelevant outcomes. In four of these, $\mathrm{N}$-acetylcysteine was used as a mucolytic for lung disease in CF, rather than for the prevention of DIOS (Baran 1980; Dietzsch 1980; Gotz 1980; Howatt 1966). In the fifth trial, N-acetylcysteine was used to improve malabsorption in $\mathrm{CF}$ rather than for the prevention of DIOS (Mitchell 1981). A further trial was excluded because the intervention was for the treatment rather than the prevention of DIOS (Dalzell 1992). The final trial was excluded because it was not randomised (O'Brien 2011).

\section{Risk of bias in included studies}

The risk of bias summary is shown in Figure 2, please also see the risk of bias table (Characteristics of included studies). 
Figure 2. Risk of bias summary: review authors' judgements about each risk of bias item for each included study.

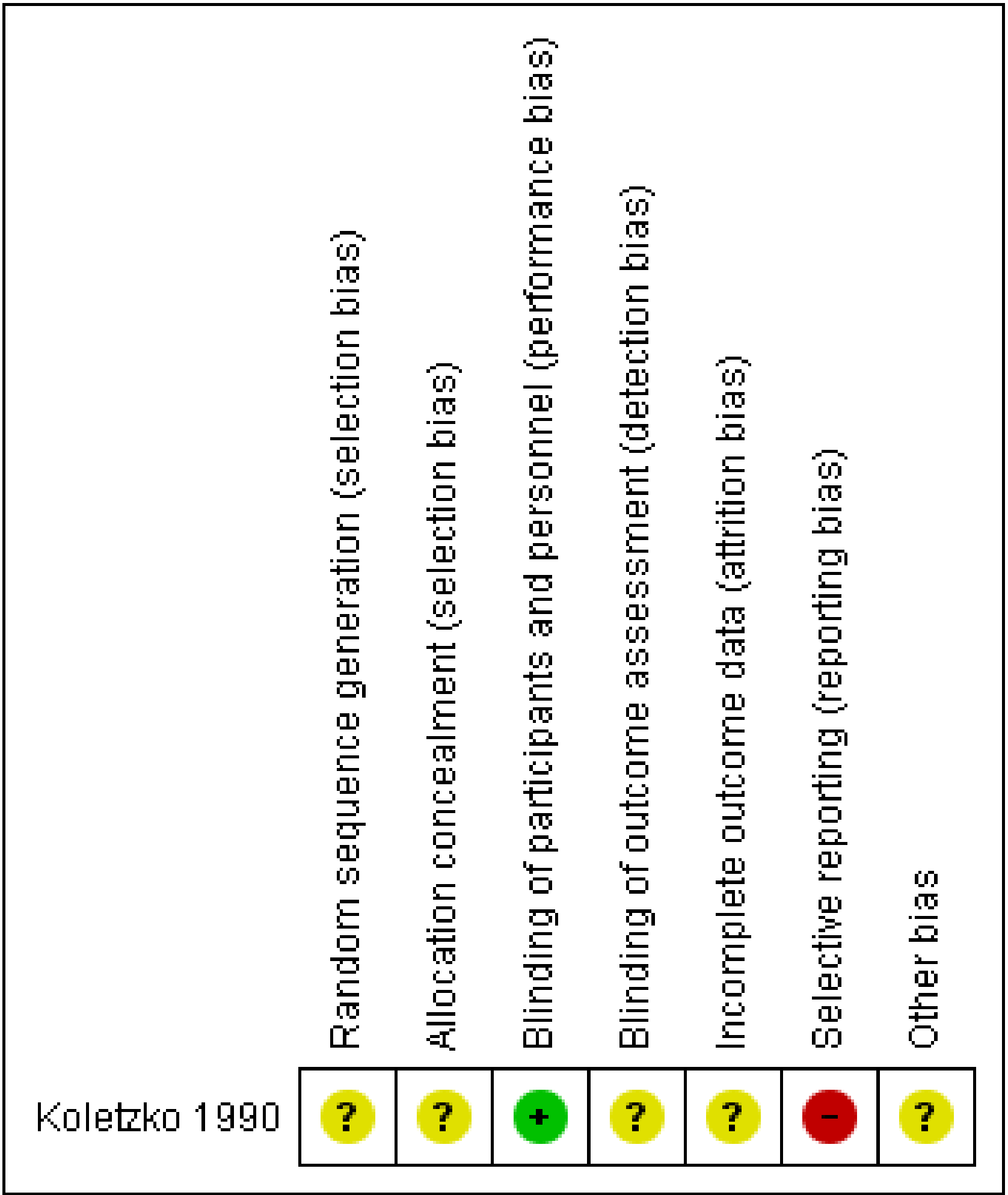

\section{Allocation}

The trial stated that participants were randomly allocated to start treatment with either cisapride or placebo, but did not specify how the sequence was generated or the method used for concealment of allocation. We therefore ranked the study as having an unclear risk of bias for these categories. 


\section{Blinding}

Participants and personnel were blinded and the placebo was identical in taste and appearance to cisapride. For this category, we ranked the trial as having a low risk of bias.

For the blinding of outcome assessors, the overall risk of bias was unclear. Only three outcomes stated that the investigators were blinded. The first of these was gastrointestinal symptom scores, where the blinded participants acted as their own assessors. Second was for the assessment of supine abdominal radiographs, where a paediatric radiologist judged these in a blind fashion. Third was for the assessment of nutritional intake and stool collection, where the blinded participants recorded their own intake and investigators also worked in a blind fashion. For the other outcomes, the risk of bias was unclear. Although blinded participants scored their own global symptoms, physicians assessed them too and we could not make the assumption that the physicians were blinded. There was no mention of blinding for the other outcomes in the trial: anthropometric measurements, number of hospital admissions, pulmonary function and frequency of pulmonary infections, laboratory tests, abdominal circumference and intestinal lavage therapy.

\section{Incomplete outcome data}

There were no missing data for gastrointestinal symptom scores and global symptom scores, both of which were outcomes included in the review. However, adverse effects, number of hospital admissions and radiological signs of DIOS (also outcomes included in the review) had missing numerical data, despite the investigators stating that they had been measured. For this reason, we ranked this category as having an unclear risk of bias.

Outcomes not included in the review that also had missing numerical data were anthropometric measurements, pulmonary function, frequency of pulmonary exacerbations and laboratory values. For nutritional intake and stool losses, only 10 out of 17 participants were represented in the results. This was because three participants had refused to perform quantitative food intake protocols and stool collections and four participants were excluded due to incomplete or inaccurate food records or stool collections. Although the investigators gave reasons for the missing data, there was no mention of an ITT analysis.

\section{Selective reporting}

We did not have access to the protocol, so could not compare the list of outcomes in the protocol with the results reported in the trial. However, we compared the outcomes listed in the methods section with the outcomes reported in the results section.

The investigators stated that they would calculate the difference in weight and percentage of ideal weight for height during the two periods, using t tests for comparison. However, these changes and results of $t$ tests were not reported in the results. Pulmonary function testing and radiological findings were measured at baseline and after six months, but the results were unreported. Anthropometric measurements (e.g. mid-arm circumference and skin fold thickness), physical examination findings, number of hospital admissions and frequency of pulmonary infections were measured every three months but insufficiently reported.
Laboratory test results (blood and urine analysis) were also measured but not reported.

Due to multiple incidences of selective reporting, we ranked the trial as having a high risk of bias for this category.

\section{Other potential sources of bias}

There was insufficient information to judge whether there was a risk of bias from other sources. In terms of publication bias, the pharmaceutical company, Janssen Pharmaceutica Incorporated, supported the trial. There was no evidence to suggest that they had any part in sponsorship or funding but this was not explicitly stated. There was also no indication to suggest conflicts of interest from the authors but again, this was not explicitly stated. Due to insufficient information regarding other sources of bias in the trial, we rated this category as having an unclear risk of bias.

\section{Effects of interventions}

See: Summary of findings for the main comparison Cisapride compared to placebo for preventing distal intestinal obstruction syndrome (DIOS) in cystic fibrosis

\section{Oral cisapride versus placebo}

Please also see Summary of findings for the main comparison.

\section{Primary outcomes}

\section{Complete or incomplete DIOS}

We pre-specified that this review outcome was to be diagnosed either clinically (e.g. abdominal masses, or distension or pain) or radiologically (e.g. dilated bowel or faecal mass). The radiological diagnosis of DIOS was measured in the included trial using a scoring system for the total radiological severity and severity of each criterion (e.g. degree and distribution of faecal retention, presence of bubbly granularity in the right iliac fossa, degree of small bowel dilatation and nodularity of the intestinal mucosa). However, the numerical data were not reported in the results. The investigators stated that there was no significant difference between cisapride and placebo (very low-quality evidence) (Koletzko 1990).

\section{Adverse effects from treatment}

Participants were interviewed for adverse effects every three months. The trial reported that no adverse effects were noted (very low-quality evidence) (Koletzko 1990).

\section{Secondary outcomes}

\section{Time to hospital admission}

This outcome was not assessed or reported in the trial either for all causes or due to DIOS (Koletzko 1990).

\section{Participant-reported QoL scores}

This outcome was not assessed or reported in the trial (Koletzko 1990).

\section{Participant-reported symptom scores}

Two different symptom scores were assessed and reported by participants in this trial: gastrointestinal symptoms and global symptoms. 
For gastrointestinal symptoms, a lower score signified a better result. Participants scored the severity and frequency of 10 different gastrointestinal symptoms and total gastrointestinal symptoms at three-monthly intervals. The trial reported results for a sixmonth period, where the scores ranged from 2 to 20 for individual symptoms and 20 to 100 for the total gastrointestinal symptom score (Koletzko 1990).

Due to the way the data were presented, we calculated the MD and standard error (SE) in order to analyse the average symptom score using the generic inverse variance (GIV). We used the fixedeffect model because there was a single trial in the review. The total gastrointestinal symptom score was statistically significant in favour of cisapride over the placebo at six months, MD -7.60 (95\% $\mathrm{Cl}-14.73$ to -0.47 ) (very low-quality evidence) (Analysis 1.1).

Individual symptom scores of interest were abdominal distension and abdominal pain, as they related to the symptoms of DIOS. There was no significant difference between cisapride and placebo for abdominal pain or abdominal distension at six months (very low-quality evidence). Abdominal pain showed a MD of $-0.4(95 \% \mathrm{Cl}$ -2.05 to 1.25 ) (Analysis 1.2) and abdominal distension showed a MD of -0.90 (95\% Cl-2.39 to 0.59 ) (Analysis 1.3).

Participants also recorded global symptom scores at the end of each six-month period (i.e. after the full course of either cisapride or placebo). The data were reported as the number of participants who fell into three categories: those who felt better, the same or worse with the treatment. Due to the way in which the data were reported, we were unable to measure this outcome as continuous or dichotomous. We presented the data in a simple table on to show the numbers of participants for each category. The trial reported that the results favoured cisapride and were statistically significant $(\mathrm{P}<0.05)$ (Table 1$)$

\section{Tolerability}

This outcome was not assessed or reported in the trial (Koletzko 1990).

\section{DISCUSSION}

\section{Summary of main results}

There was only one trial included in this review comparing cisapride to placebo for the prevention of DIOS (Koletzko 1990). There were no significant differences between cisapride and placebo for radiological diagnosis of DIOS and no side effects noted in either group. The included trial did not report on hospital admissions. The results found that cisapride improved the total gastrointestinal symptoms for participants during the trial period. Participants reported generally feeling better when taking cisapride and those with worse symptom scores benefited most from the drug. There were no significant differences between cisapride and placebo for individual symptom scores that were relevant to the review, such as abdominal distension and abdominal pain.

\section{Overall completeness and applicability of evidence}

As there was only one comparison containing one trial in the review (cisapride versus placebo), we could not perform a meta-analysis of the data and we were also unable compare the relative efficacies, safety and adverse effects of different laxative agents as we had intended. The evidence for preventing DIOS is only based on one low-quality trial that was conducted nearly 30 years ago with a drug that can no longer be prescribed in several countries, including the UK (Ferriman 2000). Therefore, the findings are very limited, irrelevant and largely inapplicable, demonstrating the huge lack of evidence for the prevention of DIOS in CF.

\section{Outcome measures}

We were unable to report several important outcome measures that we had specified in the protocol. Our primary outcome (diagnosis of complete or incomplete DIOS) was not fully reported by the investigators in a way that allowed us to analyse the data. The trial also stated that there were no adverse effects from treatment (our second primary outcome), but did not expand on this. Furthermore, the trial failed to assess most of our secondary outcomes, such as time to hospital admission, participant-reported QoL scores and tolerability. While the trial did report the number of hospital admissions, they did not report the time to the admission or specify the reason for each hospital admission. However, the trial did assess and report participant-reported symptom scores (a further secondary outcome) using gastrointestinal symptom scores and global symptom scores.

\section{Participants}

The 17 participants (both children and adults) in the included trial all had a diagnosis of CF; however, the population size was very small, which limited the precision of the effect estimates. In addition, the age range only included older children and young adults. There were no findings to demonstrate the safety and efficacy of cisapride in people with CF of other age ranges. There were 12 male participants compared to only five female participants, which could have unfairly affected the results. In 2016, Munck found that recurrent DIOS was more common in females (75\% in females compared to $52 \%$ in males, $P=0.04$ ) (Munck 2016). If this is the case, the results for the radiological diagnosis of DIOS may not be accurate, since the data mostly represents male participants.

\section{Intervention}

Cisapride is typically used a gastro-prokinetic agent and it not a typical laxative that we would have expected to be included in the review. Furthermore, cisapride has since been withdrawn from the UK market due to its cardiac side effects. Therefore, the drug has no applicability in clinical practice unless it can be re-approved for license (Ferriman 2000).

\section{Quality of the evidence}

The summary of findings table provides information about the quality of evidence in the review; the quality of evidence of the included trial is very low (Summary of findings for the main comparison).

\section{Strengths}

The design of a cross-over trial means that participants act as their own controls, which eliminates clinical differences between the two treatment arms. The investigators on the included trial also considered and eliminated the potential carry-over treatment effect that could occur with this trial design. As the trial measured the mean and SD for both the active drug and placebo, we were able to carry out a paired analysis using the GIV method. Certain domains of the risk of bias tool demonstrated high-quality trial 
design, such as the blinding of participants and personnel. Some outcomes also reported blinding of the assessors, e.g. supine abdominal radiography.

\section{Weaknesses}

There was only one included trial with a small number of participants, which led us to downgrade the evidence one level for precision. The trial was also conducted almost 30 years ago, so its results have limited relevance today. We could not assess the level of inconsistency in the review; there was only one trial included and therefore it was not possible to assess heterogeneity.

The included trial was judged to have an unclear risk of bias across most of the domains, mainly because the report did not provide sufficient information for an assessment. The most prominent areas of unclear bias were: random sequence generation, allocation concealment and incomplete outcome data.

For the outcomes presented in the summary of findings table, we downgraded the evidence quality for unclear methods in sequence generation and allocation concealment. There was also a risk of selective reporting for a number of outcomes including the radiological diagnosis of DIOS (for which the investigators did not fully report the results), pulmonary function, nutritional intake and stool losses (not presented in this review). We could not find a valid reason for why the investigators fully reported some results but not others. The trial gave reasons for some missing data and we judged that it was missing at random, but the investigators did not (to our knowledge) conduct an intention-to-treat analysis. Consequently, there was a high risk of selective reporting bias across the whole trial.

We were unable to produce a funnel plot to test for publication bias because we only included one trial in the review. We could not assume that one trial meant that there was no risk of publication bias, but equally did not strongly suspect this. We therefore rated publication bias as 'undetected'.

\section{Potential biases in the review process}

During the process of trial screening, data collection and data extraction, there was a low risk of bias. Two authors independently screened the trials and resolved any disputes mainly by discussion, but with the third author acting as an external arbiter if needed.

\section{Agreements and disagreements with other studies or reviews}

The included trial was published in 1990 when cisapride was a fully licensed drug in the UK (Koletzko 1990). It was commonly used to treat gastric and digestive disorders in children and adults. However in July 2000, the Medicines Control Agency suspended the use of cisapride in the UK due to the rare but serious cardiac effects associated with the drug (Ferriman 2000). These effects were associated with ventricular arrhythmias and in some cases, sudden death. Between 1988 and 2000, the UK received reports of 60 adverse cardiovascular reactions of the drug, five of which resulted in death. Worldwide, there were 125 fatal reactions to cisapride, which led to many other countries suspending the marketing for the drug, e.g. USA, Canada and Germany.

\section{AUTHORS' CONCLUSIONS}

\section{Implications for practice}

The results of this Cochrane Review signal that there is no evidencebase for the prevention of distal intestinal obstruction syndrome (DIOS) in clinical practice. Conversely, there are a high number of randomised controlled trials on the respiratory complications in cystic fibrosis (CF). This is likely the case because people with CF predominantly suffer from respiratory complications and are most likely to die from respiratory failure. However, many complications in CF affect the overall health of the individual. If gastrointestinal problems such as constipation and DIOS are not managed effectively, they may worsen the individual's quality of life, nutritional status, mobility, and ultimately make them more susceptible to respiratory infections. It is therefore imperative that these problems are placed at a higher level of importance.

\section{Implications for research}

As there was only one low-quality, cross-over trial eligible for inclusion in this Cochrane Review, it is evident that there is a great need for more research in this area. Future trials should include larger numbers of participants for more precision and be designed as randomised placebo-controlled trials in order to provide robust evidence. There is reliable evidence to support the use of patient-reported outcome measures (PROMs) in healthcare. A 2013 systematic review demonstrated that effective PROMs improve patient-provider communication, patient satisfaction, the monitoring of treatment response and the detection of unrecognised problems (Chen 2013). In particular, with very little research on gastrointestinal problems in CF, there is no consensus for the best outcome measures to use in gastrointestinal-focused trials. This was outlined in the 2017 James Lind Alliance Priority Setting Partnership in CF, a partnership between people with CF and healthcare providers (Rowbotham 2017). The partnership reached a consensus for a list of the 10 most important research priorities in CF, the second of which was "How can we relieve gastro-intestinal (GI) symptoms, such as stomach pain, bloating and nausea in people with CF?". This research priority emphasises the lack of PROMs for gastrointestinal problems in CF. Any future research would be significantly improved by ensuring agreed consensus on validated gastrointestinal outcome measures for symptoms.

The pathophysiology of DIOS is multifactorial. It is highly likely that there will be more than one successful strategy for preventing it. These include prokinetics, mucolytic agents and drugs or treatments which restore CFTR function.

Following on from the limited findings of this review, it would be very interesting to look at the role of prokinetic agents for preventing or treating DIOS. Although cisapride has been removed from the UK drug market amongst others, there are many other types of prokinetic agents such as domperidone and metoclopramide, as well as antibiotics with prokinetic properties such as erythromycin and azithromycin. Azithromycin is commonly used for respiratory complications in CF, so it would be interesting to see whether the incidence of DIOS in people taking azithromycin is significantly different to those not taking it. An observational study using data from the UK CF Data Registry could help obtain this information. There are various factors that may affect the incidence of DIOS; those who take regular azithromycin arguably 
have a lower standard of health and may be more likely to suffer from gastrointestinal complications, such as DIOS. However, these types of factors could be accounted for in subgroup analyses, e.g. grouping individuals according to their lung function (force expiratory volume in one second $\left(\mathrm{FEV}_{1}\right) \%$ predicted). In summary, the lack of evidence on this subject has opened up various avenues for further research. After this Cochrane Review has identified the potential use of prokinetic agents for the prevention of DIOS (albeit in a very limited way), we believe the next logical step is to further investigate their role in the management of DIOS.

We have not found evidence of mucolytic agents used for the prevention of DIOS, but we know that these are used in clinical practice. It would be helpful to look at the effectiveness and safety of mucolytic agents in a randomised controlled trial for the prevention of DIOS.

CFTR potentiators and gene therapies are a growing area of CF medicine. Ivacaftor (a CFTR potentiator) is already licensed for the G551D mutation in CF due to its significant and sustained improvements on lung function (Ramsey 2011). The combination of lumacaftor and ivacaftor (marketed as Orkambi ${ }^{\circledR}$ ) has also shown significant improvements in lung function for the most common mutation, homozygous Phe508del (US FDA 2016). It it likely that the gastrointestinal symptoms will also be improved with these agents, as they target the CFTR channel itself. They have an impact on CFTR in the pancreatic ducts, hence reducing mucus viscosity and overproduction. These agents may therefore have a positive impact on the rate of DIOS episodes. Newer gene therapies, specifically lentiviral vector gene therapy, have also shown promise in earlier studies (Alton 2017). It would be beneficial to conduct an observational study to further investigate the role of these agents in the prevention of DIOS.

Aside from exploring the use of these different agents (prokinetic laxatives, prosecretory laxatives, mucolytics and CFTR potentiators) in individual trials, it would be more robust to conduct a randomised controlled trial that could compare the different types of laxatives and CFTR potentiators. It would compare current regimens, and then standard of care to newly-released agents, either novel laxatives (prosecretory or prokinetic) or CFTR-targeted medicines, for the prevention of DIOS.

\section{ACKNOWLEDGEMENTS}

This project was supported by the National Institute for Health Research, via Cochrane Infrastructure funding to the Cochrane Cystic Fibrosis and Genetic Disorders Group. The views and opinions expressed therein are those of the authors and do not necessarily reflect those of the Systematic Reviews Programme, NIHR, NHS or the Department of Health.

We would like to thank Nikki Jahnke (Managing Editor at the Cochrane Cystic Fibrosis and Genetic Disorders Group) for her support and guidance throughout this review. 


\section{RE F E R E N C E S}

\section{References to studies included in this review}

Koletzko 1990 \{published data only\}

Koletzko S, Corey M, Ellis L, Spino M, Durie P. Effects of cisapride in patients with cystic fibrosis (CF) and chronic distal intestinal obstruction syndrome (DIOS). Pediatric Pulmonology 1989;Suppl 4:138. [CFGD Register: GN15a]

* Koletzko S, Corey M, Ellis L, Spino M, Stringer DA, Durie PR. Effects of cisapride in patients with cystic fibrosis and distal intestinal obstruction syndrome. Journal of Pediatrics 1990;117(5):815-22. [CFGD Register: GN15b]

\section{References to studies excluded from this review}

\section{Baran 1980 \{published data only\}}

Baran D. Mucolytic treatment in cystic fibrosis. Double-blind clinical trial with oral acetylcysteine and placebo. European Journal of Respiratory Diseases 1980;61:134.

\section{Dalzell 1992 \{published data only\}}

Dalzell AM, Heaf DP. High dose pancreatic enzymes in distal intestinal obstruction syndrome. Paediatric Research Society Meeting 1992, issue 149. [CFGD Register: GN170]

\section{Dietzsch 1980 \{published data only\}}

Dietzsch HJ, Berger G, Gottschalk B. Results of oral acetylcysteine therapy in children with cystic fibrosis. European Journal of Respiratory Diseases 1980;61:135.

\section{Gotz 1980 \{published data only\}}

Gotz M, Kraemer R, Kerrebijn KF, Popow, C. Oral acetylcysteine in cystic fibrosis. A co-operative study. European Journal of Respiratory Diseases. Supplement 1980;111:122-6.

\section{Howatt 1966 \{published data only\}}

Howatt WF, DeMuth GR. A double-blind study of the use of acetylcysteine in patients with cystic fibrosis. University of Michigan Medical Center Journal 1966;32(2):82-5.

Mitchell 1981 \{published data only\}

Mitchell EA, Elliott RB. Failure of oral N-acetylcysteine to improve the malabsorption of cystic fibrosis. Australian Paediatric Journal 1981;17(3):207-8.

O'Brien 2011 \{published data only\}

O'Brien CE, Anderson PJ, Stowe CD. Lubiprostone for constipation in adults with cystic fibrosis: a pilot study. Annals of Pharmacotherapy 2011;45(9):1061-6.

\section{Additional references}

\section{Alton 2017}

Alton EWFW, Beekman JM, Boyd AC, Brand J, Carlon MS, Connolly MM, et al. Preparation for a first-in-man lentivirus trial in patients with cystic fibrosis. Thorax 2017;72(2):137-47.

\section{Anderson 1990}

Andersen $\mathrm{HO}$, Hjelt K, Waever E, Overgaard K. The age-related incidence of meconium ileus equivalent in a cystic fibrosis population:the impact of a high energy intake. Journal of Paediatric Gastroenterology and Nutrition 1990;11(3):355-60.

\section{BNFc 2016}

Joint Formulary Committee. London: BMJ Group and Pharmaceutical Press, 2016. British National Formulary for Children. Vol. 72, London: BMJ Group and Pharmaceutical Press, 2016.

\section{Boyle 2009}

Boyle G, Mounsey A, Crowell K. What is the role of prokinetics for constipation. Journal of Family Practice 2009;58(4):220.

\section{CF Foundation 2016}

CF Foundation. About Cystic Fibrosis. www.cff.org/What-is-CF/ About-Cystic-Fibrosis/ (accessed 13 April 2016).

\section{Chen 2013}

Chen J, Ou L, Hollis S. A systematic review of the impact of routine collection of patient reported outcome measures on patients, providers and health organisations in an oncologic setting. BMC Health Service Research 2013;13:211.

\section{Colombo 2011}

Colombo C, Ellemunter H, Houwen R, Munck A, Taylor C, Wilschanski M. Guidelines for the diagnosis and management of distal intestinal obstruction syndrome in cystic fibrosis patients. Journal of Cystic Fibrosis 2011;10 Suppl 2:S24-8. [DOI: 10.1016/ S1569-1993(11)60005-2]

\section{Davidson 1987}

Davidson AC, Harrison K, Steinfort CL, Geddes DM. Distal intestinal obstruction syndrome in cystic fibrosis treated by oral intestinal lavage, and a case of recurrent obstruction despite normal pancreatic function. Thorax 1987;42(7):538-41.

\section{Deeks 2011}

Deeks J, Higgins J, Altman D, editor(s) on behalf of the Cochrane Statistical Methods Group. Chapter 9 Analysing data and undertaking meta-analysis. In: Higgins JPT, Green S, editor(s). Cochrane Handbook for Systematic Reviews of Interventions Version 5.1.0 (updated March 2011). The Cochrane Collaboration, 2011. Available from cochrane-handbook.org.

\section{Dray 2004}

Dray X, Bienvenu T, Desmazes-Dufeu N, Dusser D, Marteau P, Hubert D. Distal intestinal obstruction syndrome in adults with cystic fibrosis. Clinical Gastroenterology and Hepatology 2004;2(6):498-503.

\section{Elbourne 2002}

Elbourne DR, Altman DG, Higgins JPT, Curtin F, Worthington HV, Vail A. Meta-analyses involving cross-over trials: methodological issues. International Journal of Epidemiology 2002;31(1):140-9. 


\section{Ferriman 2000}

Ferriman A. UK licence for cisapride suspended. BMJ 2000;321(7256):259.

\section{Gilljam 2003}

Gilljam M, Chaparro C, Tullis E, Chan C, Keshavjee S, Hutcheon M. Gastrointestinal complications after lung transplantation in patients with cystic fibrosis. Chest 2003;123(1):37-41.

\section{Higgins 2011}

Higgins JPT, Altman DG, Sterne JAC, editor(s) on behalf of the Cochrane Statistical Methods Group and the Cochrane Bias Methods Group. Chapter 8: Assessing risk of bias in included studies. In: Higgins JPT, Green S, editor(s). Cochrane Handbook for Systematic Reviews of Interventions. Version 5.1.0 (updated March 2011). The Cochrane Collaboration, 2011. Available from cochrane-handbook.org.

\section{Houwen 2010}

Houwen RH, van der Doef HP, Sermet I, Munck A, Hauser B, Walkowiak J, et al. Defining DIOS and constipation in cystic fibrosis with a multicentre study on the incidence, characteristics, and treatment of DIOS. Journal of Paediatric Gastroenterology and Nutrition 2010;50(1):38-42. [DOI: 10.1097/ MPG.0b013e3181a6e01d]

\section{Millar-Jones 1995}

Millar-Jones L, Goodchild MC. Cystic fibrosis, pancreatic sufficiency and distal intestinal obstruction syndrome: a report of four cases. Acta Paediatrica 1995;84(5):577-8.

\section{Moher 2009}

Moher D, Liberati A, Tetzlaff J, Altman DG. The PRISMA Group. Preferred reporting items for systematic reviews and meta-analyses: The PRISMA Statement. PLoS Medicine 2009;6(7):e1000097. [DOI: 10.1371/journal.pmed.1000097]

\section{Munck 2016}

Munck A, Corinne A, Colombo C, Kashirskaya N, Ellemunter H, Fotoulaki $\mathrm{M}$, et al on behalf of the CF/Pancreas ESPGHAN Working Group and DIOS Study Group. International prospective study of distal intestinal obstruction syndrome in cystic fibrosis: associated factors and outcome. Journal of Cystic Fibrosis 2016;15(4):531-9.

\section{NICE 2015}

National Institute for Healthcare and Excellence (NICE). Clinical knowledge summaries. Constipation in children (last revised June 2015). cks.nice.org.uk/constipation-in-children\#!scenario (accessed 03 October 2016).

\section{NICE 2017}

National Institute for Healthcare and Excellence (NICE). Clinical knowledge summaries. Constipation (last revised June 2017). https://cks.nice.org.uk/constipation\#!prescribinginfosub:2 (accessed 25 February 2018).

\section{Penketh 1987}

Penketh AR, Wise A, Mearns MB, Hodson ME, Batten JC. Cystic fibrosis in adolescents and adults. Thorax 1987;42(7):526-32.

\section{Ramsey 2011}

Ramsey BW, Davies J, McElvaney NG, Tullis E, Bell SC, Dřevínek $\mathrm{P}$, et al. A CFTR potentiator in patients with cystic fibrosis and the G551D mutation. New England Journal of Medicine 2011;365(18):1663-72.

\section{RevMan 2014 [Computer program]}

The Nordic Cochrane Centre, The Cochrane Collaboration. Review Manager (RevMan). Version 5.3. Copenhagen: The Nordic Cochrane Centre, The Cochrane Collaboration, 2014.

\section{Rowbotham 2017}

Rowbotham NJ, Smith S, Leighton PA, Rayner OC, Gathercole K, Elliott ZC, et al. The top 10 research priorities in cystic fibrosis developed by a partnership between people with CF and healthcare providers. Thorax 2017;73(4):388-90. [DOI: 10.1136/ thoraxjnl-2017-210473]

\section{Rubinstein 1986}

Rubinstein S, Moss R, Lewiston N. Constipation and meconium ileus equivalent in patients with cystic fibrosis. Pediatrics 1986; Vol. 78, issue 3:473-9.

\section{Schunemann 2006}

Schunemann HJ, Fretheim A, Oxman AD. Improving the use of research evidence in guideline development: 13. Applicability, transferability and adaptation. Health Research Policy \& Systems 2006;4:25.

\section{Sterne 2011}

Sterne JAC, Egger M, Moher D, editor(s) on behalf of the Cochrane Bias Methods Group. Chapter 10: Addressing reporting biases. In: Higgins JPT, Green S, editor(s). Cochrane Handbook for Systematic Reviews of Interventions Version 5.1.0 (updated March 2011). The Cochrane Collaboration, 2011. Available from cochrane-handbook.org.

\section{Tobias 2011}

Tobias ES, Connor M, Ferguson-Smith M. Essential Medical Genetics. 6th Edition. Hoboken, NJ, USA: Wiley-Blackwell, 2011.

\section{Tonni 1996}

Tonni M. Recent advances in the pharmacology of gastrointestinal prokinetics. Pharmacological Research 1996;33(4-5):217-26

\section{Tuldahar 1999}

Tuladhar R, Daftary A, Patole SK, Whitehall JS. Oral gastrografin in neonates: a note of caution. International Journal of Clinical Practice 1999;53(7):565.

\section{US FDA 2016}

US Food, Drug Administration. Orkambi ${ }^{\mathrm{TM}}$ (lumacaftor/ivacaftor) [prescribinginformation]. www.fda.gov/Safety/MedWatch/ SafetyInformation/ucm505856.htm (accessed before 18 April 2018).

\section{Van der Doef 2010}

Van der Doef HPJ, Kokke FTM, Beek FJA, Woestenenk JW, Froeling SP, Houwen RHJ. Constipation in paediatric cystic 
fibrosis patients: an underestimated medical condition. Journal of Cystic Fibrosis 2010;9(1):59-63.

\section{Van der Doef 2011}

Van der Doef HP, Kokke FT, van der Ent CK, Houwen RH. Intestinal obstruction syndromes in cystic fibrosis: meconium ileus, distal intestinal obstruction syndrome, and constipation. Current Gastroenterology Reports 2011;13(3):265-70. [DOI: 10.1007/s11894-011-0185-9]

* Indicates the major publication for the study

\section{CHARACTERISTICS OF STUDIES}

Characteristics of included studies [ordered by study ID]

\section{Koletzko 1990}

\section{Methods}

Study design: randomised controlled trial, double-blind placebo-controlled

Study grouping: cross-over, each arm lasted for 6 months

Carry-over treatment effect accounted for: yes. Measurements were recorded twice for each 6month period. Investigators took an average of the 2 measurements, they then discarded the measurements from the first 3 months, to account for any cumulative effect of the drug. There was no formal washout period

\section{Participants}

Inclusion criteria: diagnosis of CF (by sweat test $>60 \mathrm{mmol} / \mathrm{L}$ ); 1 or more episodes of DIOS in preceding 12 months (based on clinical or radiological evidence and on successful treatment with intestinal lavage, oral $\mathrm{N}$-acetylcysteine or both)

Exclusion criteria: peptic ulcer disease, inflammatory bowel disease, anatomic intestinal obstruction, serious cardiovascular, neurological, renal or hepatic disease, severe pulmonary dysfunction (maximal mid-expiration flow rate $<25 \%$ normal) pregnancy, regular use of metoclopramide, domperidone or anticholinergic drug

Pre-treatment: no significant differences in the clinical characteristics of 2 groups (cross-over)

\section{Baseline characteristics}

Age, mean (SD), range: 21.0 (5.9) years, 12.9 to 34.9 years old

Mean (SD) duration of DIOS symptoms: 4.2 (3.1) years

Mean (SD) height percentile: 40.6 (26.3)

Mean (SD) weight percentile: 40.3 (23.2)

Mean (SD) weight for height: 100.3 (9.5) \% of ideal

Mean (SD) FVC (\% predicted): 80.9 (23.4)\%

Mean (SD) FEV 1 (\% predicted): $78.2(28.1) \%$

Interventions

Cisapride: oral tablets $7.5 \mathrm{mg} 3$ times daily (before meals) for participants weighing between $40 \mathrm{~kg}$ and $50 \mathrm{~kg}, 10 \mathrm{mg} 3$ times daily (before meals) for participants weighing over $50 \mathrm{~kg}$

Placebo (identical in appearance to active treatment): oral tablets $7.5 \mathrm{mg} 3$ times daily (before meals) for participants weighing between $40 \mathrm{~kg}$ and $50 \mathrm{~kg}, 10 \mathrm{mg} 3$ times daily (before meals) for participants weighing over $50 \mathrm{~kg}$

Outcomes

Gastrointestinal symptoms: a total of 10 symptoms scored 1 to 10 (heartburn, flatulence, regurgitation, fullness, abdominal distension, abdominal pain, diarrhoea, nausea, vomiting, anorexia) and also added to give a total score (20 to 100) where a lower score is better; severity (none, not limiting daily activities, limits daily activities, daily activities not possible) and frequency (never, less than once a week, more than once a week or daily) of symptoms recorded and accounted for in final score for each item

Anthrompometric measurements: mid-arm circumference; skin fold thickness; change in weight; abdominal circumference 
Alteration in global symptoms: measured by both physician and participant, from 'worse' to 'symptom-free' using scores from -1 to +3 (higher is better)

Pulmonary function: $\mathrm{FEV}_{1} \%$ predicted, frequency of pulmonary exacerbations, number of hospital admissions

Nutrient intake: calories ( $\mathrm{kcal} / \mathrm{day})$, fat ( $\mathrm{mmol} / \mathrm{day})$

Stool composition: stool weight (g/day), faecal water content (\%), faecal fat (\%), calories malabsorbed (\%), faecal bile acids (mmol/day), faecal chymotrypsin (10 x 300 units/day)

\section{Supine abdominal radiography}

Intestinal lavage therapy

Sponsorship source: supported by Janssen Pharmaeutica Inc., Canada. No sponsorship mentioned

Country: Canada

Setting: single centre (tertiary centre) at the University of Toronto and the research institute at the Hospital for Sick Children, Toronto

Comments: approved by the human subjects review committee of the Hospital for Sick Children, Toronto. Janssen Pharmaceutica supplied cisapride and placebo tablets

Contact author's name: Sibylle Koletzko

Institution: Children's Hospital, Heinrich Heine University, Dusseldorf, Federal Republic of Germany

Email: Sibylle.Koletzko@med.uni-muenchen.de

Address: Lindwurmstraße 4, 80337 München, Germany

The authors did not declare any conflicts of interest

Notes

\section{Risk of bias}

\begin{tabular}{lll}
\hline Bias & Authors' judgement & Support for judgement \\
\hline $\begin{array}{l}\text { Random sequence genera- } \\
\text { tion (selection bias) }\end{array}$ & Unclear risk & $\begin{array}{l}\text { Random allocation stated but no specific sequence generation methodology } \\
\text { described. }\end{array}$ \\
\hline $\begin{array}{l}\text { Allocation concealment } \\
\text { (selection bias) }\end{array}$ & Unclear risk & No method described for concealment of allocation. \\
\hline $\begin{array}{l}\text { Blinding of participants } \\
\text { and personnel (perfor- } \\
\text { mance bias) } \\
\text { All outcomes }\end{array}$ & Low risk & $\begin{array}{l}\text { "Double-blind" trial. Placebo tablets were identical in taste and appearance to } \\
\text { cisapride. }\end{array}$ \\
\hline
\end{tabular}

Blinding of outcome as- Unclear risk sessment (detection bias)
Only 3 outcomes stated that the investigators were blinded.

1. Gastrointestinal symptom scores - the blinded participants acted as their own assessors.

2. Assessment of supine abdominal radiographs - a paediatric radiologist judged these in a blind fashion.

3. Assessment of nutritional intake and stool collection - the blinded participants recorded their own intake and investigators also worked in a blind fashion. 
Although blinded participants scored their own global symptoms, physicians assessed them too and we could not make the assumption that the physicians were blinded.

There was no mention of blinding for the other outcomes: anthropometric measurements, number of hospital admissions, pulmonary function and frequency of pulmonary infections, laboratory tests, abdominal circumference and intestinal lavage therapy.

Incomplete outcome data Unclear risk (attrition bias)

All outcomes
No missing data and outcomes fully reported: gastrointestinal symptom scores, global symptom scores and intestinal lavage therapy

Insufficient information to judge whether outcome data are missing: anthropometric measurements, adverse effects, pulmonary function, frequency of pulmonary exacerbations, number of hospital admissions, radiological signs of DIOS and laboratory values

Data missing: for nutritional intake and stool losses, only present data for $10 / 17$ participants, reasons given for missing data but this would have had a big impact on the effect size, since the sample size was small anyway; no mention of ITT analysis, no mention of treating per protocol

No access to trial protocol so not possible to compare planned outcomes to outcomes reported in paper.

Anthropometric measurements: stated in the methods section that "at the end of each treatment period, difference weight and percentage of ideal weight for height, as well as the difference in weight change during the two periods, were compared by means of $t$ tests", however, these changes and results of $t$ tests were not specifically reported in the results, incomplete report means it can not be entered into a meta- analysis.

Pulmonary function testing and $\mathrm{X}$-ray findings: although supposed to be measured "at the end of baseline and 6 month periods" were reported incompletely so cannot be entered into a meta-analysis.

Cisapride levels: in the methods section, it specified that they would determine cisapride levels "at the end of baseline and 6 month periods" but this was not reported in the results.

Number of hospital admissions: specified as an outcome measure in the methods section, but not reported in the results.

Laboratory test results (blood and urine analysis): incompletely reported so that they could not be entered into a meta-analysis.

None known, but cannot be sure, there is no known sponsorship source but the trial was described as being supported by Janssen Pharmaceuticals.

CF: cystic fibrosis

DIOS: distal intestinal obstruction syndrome

$\mathrm{FEV}_{1}$ : forced expiratory volume at one second

FVC: forced vital capacity

ITT: intention-to-treat analysis

Characteristics of excluded studies [ordered by study ID] 


\begin{tabular}{ll}
\hline Study & Reason for exclusion \\
\hline Baran 1980 & $\begin{array}{l}\text { Inappropriate indication: N-acetylcysteine used for respiratory complications rather than for pre- } \\
\text { venting DIOS }\end{array}$ \\
\hline Dalzell 1992 & $\begin{array}{l}\text { Inappropriate interventions: pancreatic enzyme therapy used for treatment of DIOS instead of pre- } \\
\text { vention of DIOS }\end{array}$ \\
\hline Dietzsch 1980 & $\begin{array}{l}\text { Inappropriate indication: N-acetylcysteine used for respiratory complications rather than for pre- } \\
\text { venting DIOS }\end{array}$ \\
\hline Gotz 1980 & $\begin{array}{l}\text { Inappropriate indication: N-acetylcysteine used for respiratory complications rather than for pre- } \\
\text { venting DIOS }\end{array}$ \\
\hline Howatt 1966 & $\begin{array}{l}\text { Inappropriate indication: N-acetylcysteine used for respiratory complications rather than for pre- } \\
\text { venting DIOS }\end{array}$ \\
\hline Mitchell 1981 & $\begin{array}{l}\text { Inappropriate indication: N-acetylcysteine used for malabsorption in CF rather than for preventing } \\
\text { DIOS }\end{array}$ \\
\hline N'Brien 2011 & Non-randomised and open-label design \\
\hline
\end{tabular}

CF: cystic fibrosis

DIOS: distal intestinal obstruction syndrome

\section{DATA AND ANALYSES}

\section{Comparison 1. Cisapride versus placebo}

\begin{tabular}{lllll}
\hline $\begin{array}{l}\text { Outcome or subgroup ti- } \\
\text { tle }\end{array}$ & No. of studies & $\begin{array}{l}\text { No. of partici- } \\
\text { pants }\end{array}$ & Statistical method & Effect size \\
\hline $\begin{array}{l}1 \text { Total gastrointestinal } \\
\text { symptoms }\end{array}$ & 1 & Mean Difference (Fixed, 95\% Cl) & Totals not selected \\
\hline 1.1 At 6 months & 1 & Mean Difference (Fixed, 95\% Cl) & $0.0[0.0,0.0]$ \\
\hline 2 Abdominal pain & 1 & Mean Difference (Fixed, 95\% Cl) & Totals not selected \\
\hline 2.1 At 6 months & 1 & Mean Difference (Fixed, 95\% Cl) & $0.0[0.0,0.0]$ \\
\hline 3 Abdominal distension & 1 & Mean Difference (Fixed, 95\% Cl) & Totals not selected \\
\hline 3.1 At 6 months & 1 & Mean Difference (Fixed, 95\% Cl) & $0.0[0.0,0.0]$ \\
\hline
\end{tabular}

Analysis 1.1. Comparison 1 Cisapride versus placebo, Outcome 1 Total gastrointestinal symptoms.

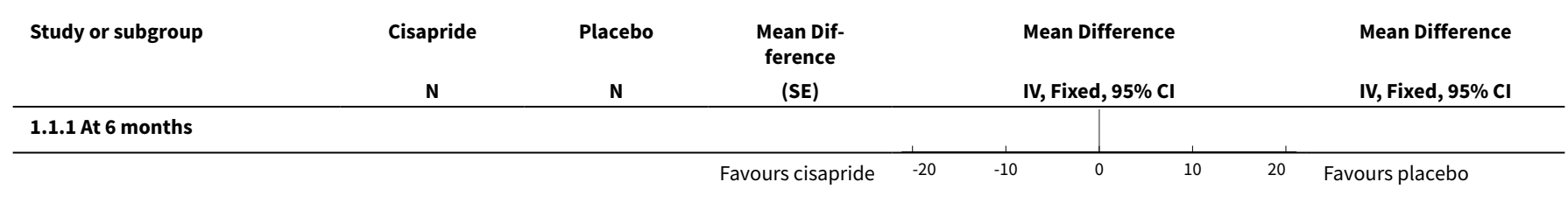




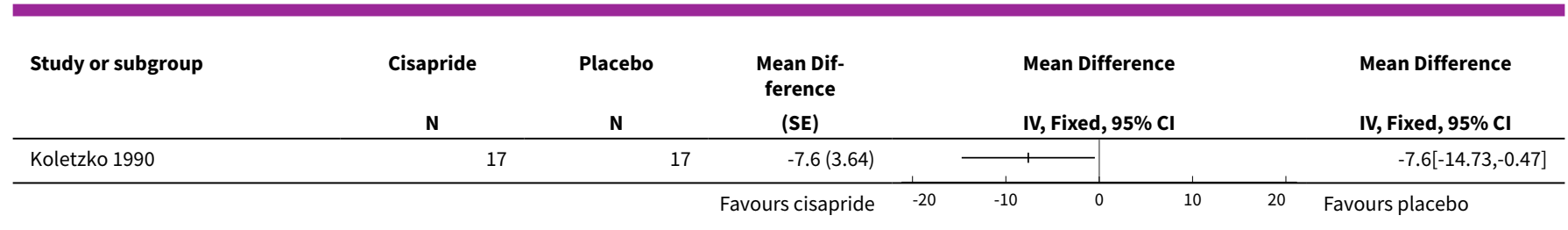

Analysis 1.2. Comparison 1 Cisapride versus placebo, Outcome 2 Abdominal pain.

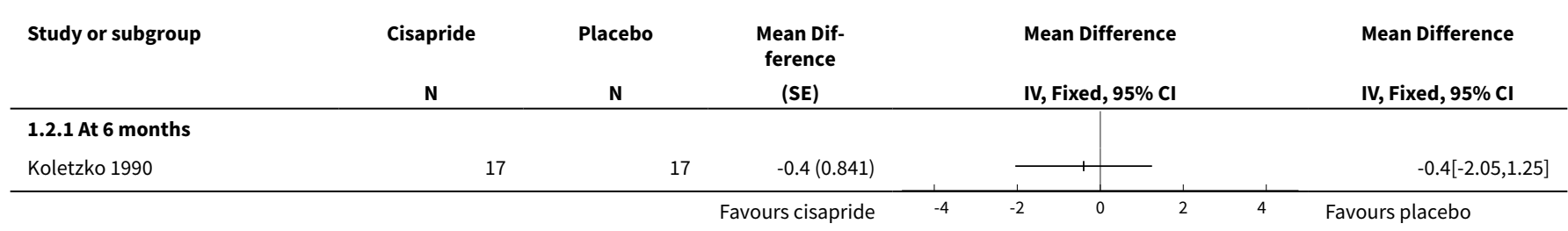

Analysis 1.3. Comparison 1 Cisapride versus placebo, Outcome 3 Abdominal distension.

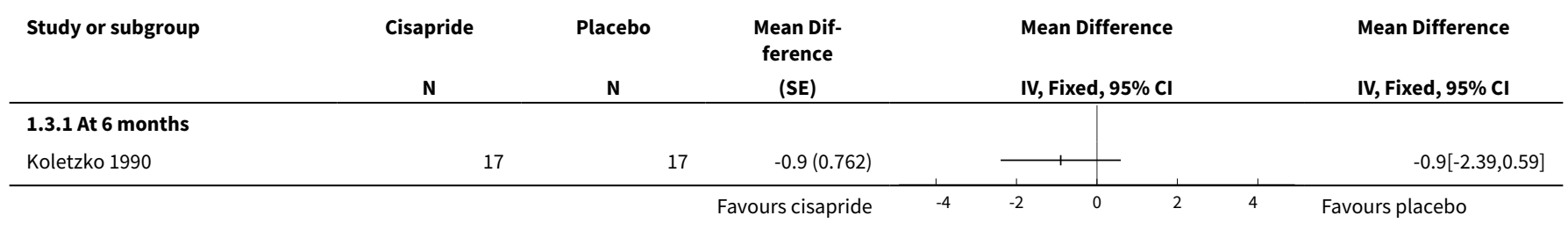

\section{ADDITIONAL TABLES}

Table 1. Alterations in global symptoms

\begin{tabular}{llllc}
\hline Intervention & Total number of participants & Felt better & Felt the same & Felt worse \\
\hline Cisapride & 17 & 12 & 2 & 3 \\
\hline Placebo & 17 & 3 & 2 & 12 \\
\hline
\end{tabular}

\section{APPENDICES}

\section{Appendix 1. Search strategies}

\begin{tabular}{ll}
\hline Database/Resource & Strategy \\
\hline Cochrane Central Register of & $\# 1$ Cystic Fibrosis [MeSH descriptor] \\
Controlled Trials (CENTRAL) & $\# 2$ cystic fibrosis:ti,ab \\
& $\# 3$ fibrocystic near/10 disease near/10 pancreas \\
\hline
\end{tabular}




$$
\begin{aligned}
& \text { \#4 mucoviscidos }{ }^{\star}: \text { ti,ab } \\
& \text { \#5 cystic }{ }^{\star} \text { near/10 fibros*:ti,ab } \\
& \# 6 \text { \#1 or \#2 or \#3 or \#4 or \#5 }
\end{aligned}
$$

\#7 distal intestinal obstruction syndrome*:ti,ab

\#8 dios or mie:ti,ab

\#9 Intestinal Obstruction [MeSH descriptor]

\#10 meconium ileus equivalent:ti,ab

\#11 faecal near/3 (obstruction or impact ${ }^{\star}$ ):ti,ab

\#12 Constipation [MeSH descriptor]

\#13 constipat ${ }^{\star}:$ ti,ab

\#14 laxative*:ti,ab

\#15 Laxatives [MeSH descriptor]

\#16 lactulose:ti,ab

\#17 Lactulose [MeSH descriptor]

\#18 (macrogol or polyethylene glycol ${ }^{\star}$ ):ti,ab

\#19 Polyethylene Glycols [MeSH descriptor]

\#20 movicol:ti,ab

\#21 klean*:ti,ab

\#22 diatriozate:ti,ab

\#23 gastrografin:ti,ab

\#24 senna:ti,ab

\#25 docusate:ti,ab

\#26 picosulfate:ti,ab

\#27 acetylcysteine or fibrol:ti,ab

\#28 parvolex:ti,ab

\#29 fibre:ti,ab

\#30 picosulphate:ti,ab

$\# 31 \# 7$ or \#8 or \#9 or \#10 or \#11 or \#12 or \#13 or \#14 or \#15 or \#16 or \#17 or \#18 or \#19 or \#20 or \#21 or \#22 or \#23 or \#24 or \#25 or \#26 or \#27 or \#28 or \#29\#30

\#32 \#6 and \#31

MEDLINE Ovid (1946 onwards) 1. Cystic Fibrosis/

2. cystic fibrosis.tw.

3. (fibrocystic adj10 disease adj10 pancreas).tw.

4. mucoviscidos\$.tw. 
5. (cystic\$ adj10 fibros\$).tw.

6. 1 or 2 or 3 or 4 or 5

7. "distal intestinal obstruction syndrome ${ }^{\star "} . t w$.

8. (dios or mie).tw.

9. Intestinal Obstruction/

10. meconium ileus equivalent.tw.

11. (faecal adj3 (obstruction or impact $\left.{ }^{\star}\right)$ ).tw.

12. Constipation/

13. "constipat". tw.

14. "laxative*".tw.

15. Laxatives/

16. lactulose.tw. or Lactulose/

17. (macrogol or polyethylene glycol ${ }^{\star}$ ).tw. or Polyethylene Glycols/

18. movicol.tw.

19. klean*.tw.

20. diatriozate.tw.

21. gastrografin.tw.

22. senna.tw.

23. docusate.tw.

24. bicosulfate.tw.

25. acetylcysteine or fibrol.tw.

26. parvolex.tw.

27. fibre.tw.

28. picosulphate.tw.

29.7 or 8 or 9 or 10 or 11 or 12 or 13 or 14 or 15 or 16 or 17 or 18 or 19 or 20 or 21 or 22 or 23 or 24 or 25 or 26 or 27 or 28

30.6 and 29

Embase Ovid (1974 onwards) 1. CYSTIC FIBROSIS/

2. cystic fibrosis.tw.

3. (fibrocystic adj10 disease adj10 pancreas).tw.

4. mucoviscidos\$.tw.

5. (cystic\$ adj10 fibros\$).tw.

6. 1 or 2 or 3 or 4 or 5

7. "distal intestinal obstruction syndrome*".tw. 
8. (dios or mie).tw.
9. INTESTINE OBSTRUCTION/

10. meconium ileus equivalent.tw.

11. (faecal adj3 (obstruction or impact $\left.{ }^{\star}\right)$ ).tw.

12. CONSTIPATION/

13. "constipat*".tw.

14. "laxative*".tw.

15. LAXATIVE/

16. lactulose.tw. or LACTULOSE/

17. (macrogol or polyethylene glycol $\left.{ }^{\star}\right) \cdot \mathrm{mp}, \mathrm{hw}$.

18. movicol.tw.

19. klean ${ }^{\star}$. tw.

20. diatriozate.tw.

21. gastrografin.tw.

22. senna.tw.

23. docusate.tw.

24. bicosulfate.tw.

25. acetylcysteine or fibrol.tw.

26. parvolex.tw.

27. fibre.tw.

28. picosulphate.tw.

29.7 or 8 or 9 or 10 or 11 or 12 or 13 or 14 or 15 or 16 or 17 or 18 or 19 or 20 or 21 or 22 or 23 or 24 or 25 or 26 or 27 or 28

30.6 and 29

Clinicaltrials.gov

\section{Search 1}

Search terms: laxative OR laxatives OR lactulose OR macrogol OR polyethylene OR movicol OR klean OR diatriozate OR gastrografin OR senna OR docusate OR bicosulfate OR acetylcysteine OR fibrol OR parvolex OR picosulphate OR fibre

Study type: Interventional Studies

Conditions: cystic fibrosis

\section{Search 2}

Search terms: intestinal OR DIOS OR constipation OR constipated OR faecal OR meconium Study type: Interventional Studies

Conditions: cystic fibrosis

\section{ISRCTN Registry}

\section{ADVANCED SEARCH}

Condition: cystic fibrosis 
Search 1: cystic fibrosis AND intestinal

Search 2: cystic fibrosis AND constipation

Search 3: cystic fibrosis AND faecal

Search 4: cystic fibrosis AND meconium

Search 5: mucoviscidose

ADVANCED SEARCH

Condition: cystic fibrosis

Intervention: laxative OR laxatives OR lactulose OR macrogol OR polyethylene OR movicol OR klean OR diatriozate OR gastrografin OR senna OR docusate OR bicosulfate OR acetylcysteine OR fibrol OR parvolex OR picosulphate OR fibre

Recruitment Status: All

Open Grey

(cystic fibrosis OR cf OR mucoviscidos*) AND (intestin* OR constipat* OR faecal OR meconium OR laxative* OR lactulose OR macrogol OR polyethylene OR movicol OR klean* OR diatriozate OR gastrografin OR senna OR docusate OR bicosulfate OR acetylcysteine OR fibrol OR parvolex OR picosulphate OR fibre)

\section{CONTRIBUTIONS OF AUTHORS}

\section{Roles and responsibilities}

\begin{tabular}{ll}
\hline TASK & WHO UNDERTOOK THE TASK \\
\hline Protocol stage: draft the protocol & WC \\
\hline Review stage: select which trials to include $(2+1$ arbiter $)$ & $\mathrm{JG}+\mathrm{WC}+\mathrm{FG}$ as arbiter \\
\hline Review stage: extract data from trials (2 people) & $\mathrm{JG}+\mathrm{WC}$ \\
\hline Review stage: enter data into RevMan & $\mathrm{JG}$ \\
\hline Review stage: carry out the analysis & $\mathrm{JG}+\mathrm{WC}$ \\
\hline Review stage: interpret the analysis & $\mathrm{JG}+\mathrm{WC}$ \\
\hline Review stage: draft the final review & $\mathrm{JG}+\mathrm{WC}$ \\
\hline Update stage: update the review & WC
\end{tabular}

\section{DECLARATIONS OF INTEREST}

Jessica Green declares no known potential conflict of interest.

Dr Will Carroll declares no known potential conflict of interest.

Dr Francis J Gilchrist declares no known potential conflict of interest. 


\section{SOURCES OF SUPPORT}

\section{Internal sources}

- No sources of support supplied

\section{External sources}

- National Institute for Health Research, UK.

This systematic review was supported by the National Institute for Health Research, via Cochrane Infrastructure funding to the Cochrane Cystic Fibrosis and Genetic Disorders Group.

\section{DIFFERENCES BETWEEN PROTOCOL AND REVIEW}

In the protocol, we did not list prokinetic agents as a possible treatment for distal intestinal obstruction syndrome (DIOS). However, we have now included them in the review because there is evidence in the literature for their role in treating constipation and generally increasing colonic transit and motility (Boyle 2009; Colombo 2011). As the treatment of constipation is especially important in the prevention of DIOS, we decided to include them as a possible intervention in this review.

\section{N D EX TERMS}

\section{Medical Subject Headings (MeSH)}

Cisapride [ ${ }^{\star}$ therapeutic use]; Cystic Fibrosis [ ${ }^{*}$ complications]; Gastrointestinal Agents [ ${ }^{*}$ therapeutic use]; Intestinal Obstruction [etiology] [pathology] [* prevention \& control]; Syndrome

\section{MeSH check words}

Adolescent; Adult; Humans 